\title{
EMULSION STABILISATION BY COMPLEXES OF OPPOSITELY CHARGED SYNTHETIC POLYELECTROLYTES
}

\author{
Ana Maria Bago Rodriguez, ${ }^{1}$ Bernard P. Binks ${ }^{1,}{ }^{*}$ and Tomoko Sekine ${ }^{2}$ \\ ${ }^{1}$ School of Mathematics and Physical Sciences, University of Hull, Hull, HU6 7RX, UK \\ ${ }^{2}$ Shiseido Global Innovation Center, 2-2-1 Hayabuchi, Tsuzuki-ku, Yokohama 224-8558, \\ Japan
}
Submitted to: $\quad$ Soft Matter on $13.9 .17 ; 1^{\text {st }}$ revision on $13.11 .17 ; 2^{\text {nd }}$ revision on 30.11.2017

\section{Contains ESI}

*Proofs and correspondence to: $\quad$ Prof. B.P. Binks

b.p.binks@hull.ac.uk 


\begin{abstract}
We investigate the possibility of stabilising oil-water emulsions from the polyelectrolyte complexes (PEC) obtained in mixtures of a strong cationic polyelectrolyte (poly(diallyldimethylammonium chloride), PDADMAC) and a weak anionic one (poly(acrylic acid) sodium salt, PAANa). Unlike other previous work however, both polyelectrolytes (PEL) are chosen as they are completely water-soluble and possess no surface activity when present alone over nearly all the $\mathrm{pH}$ range. In water, the effects of PEL concentration, PEL mixing ratio and $\mathrm{pH}$ on the formation of PEC are studied in detail. At low $\mathrm{pH}$ where the anionic PEL is uncharged, complex coacervation occurs in which droplets rich in both polymers are dispersed in water. At intermediate $\mathrm{pH}$, the PEC comprise a mixture of coacervate droplets and solid particles. At high $\mathrm{pH}$ where the anionic PEL is significantly charged, only complex coacervation is observed. On addition of dodecane followed by homogenisation, no stable emulsions arose from dispersions containing solid particle PEC due to either the large precursor particle aggregates or their inherent hydrophilicity. By contrast, oil-in-water emulsions stable to coalescence could be prepared from coacervate dispersions. We discuss the feasibility of the coacervate phase spreading at the oil-water interface in terms of the relevant spreading coefficients and compare the predictions with experiment for a range of oils. We encounter oils whose drops become engulfed by the coacervate phase as well as oils where no engulfing occurs.
\end{abstract}




\section{INTRODUCTION}

When mixing two polymer solutions, different types of phase separation can occur. If the polymers are incompatible (they repel each other), segregative phase separation takes place and each polymer is collected (predominantly) in a different phase. One example is the two phases formed by mixing dextran and methylcellulose in water. ${ }^{1}$ Complete miscibility appears in exceptional cases and gives rise to a homogeneous solution as in the mixture of polystyrene and poly(2,6-dimethyl-1,4-phenylene oxide). ${ }^{2}$ Finally, if the polymers show net attraction, usually through electrostatic interactions as with oppositely charged polyelectrolytes (PEL), associative phase separation is achieved. In this case, the two polymers are collected in one phase while the other phase consists almost entirely of solvent. This interaction can lead either to the formation of a complex coacervate (liquid-liquid type of phase separation) or a precipitate (solid-liquid type of phase separation). Both complexation mechanisms are driven by an increase of entropy due to the release of counter-ions initially bound to the PEL chains. Although the factors that dictate the nature of each type of associative phase separation are not fully elucidated, it is generally assumed that strong interactions between PELs results in precipitates while in coacervation the interactions are relatively weak. ${ }^{3,4}$ The distinction between these two types of phase separation is not clear in the literature and the general term polyelectrolyte complex (PEC) is adopted for both scenarios. However, optical microscopy is a simple and useful technique to distinguish between precipitation (amorphous solid particles) and complex coacervation (micron sized droplets). ${ }^{3}$

The term coacervation was coined by Bungenberg de Jong and Kruyt in 1929 and derives from the Latin words "co" (together) and "acerv" (a heap or aggregate). ${ }^{5}$ In coacervation, a dense polymer-rich phase (coacervate phase) and a very dilute polymerdeficient phase (aqueous phase) coexist. ${ }^{6}$ Coacervates are subdivided into simple and complex. In simple coacervation one macromolecule is present and the associative process is induced by the addition of a dehydrating agent such as salt or a change in temperature or $\mathrm{pH}$ of the medium. One example is the mixture of polyethylene glycol, potassium phosphate and water, in which the bottom phase is rich in salt and the top phase is rich in polymer. ${ }^{1}$ In complex systems, two oppositely charged species are involved and two immiscible liquid phases arise. The upper phase (supernatant) consists almost entirely of solvent whilst the bottom phase is a dense clear liquid phase (coacervate) concentrated in both types of macromolecule. Before reaching this steady state, a turbid mixture composed of a metastable suspension of macroion-rich coacervate droplets dispersed in solvent is present. ${ }^{6}$ Coacervates (droplets) hold higher amounts of water 
compared to precipitates (particles). Jha et al. determined the weight fraction of water in the coacervate system containing poly(acrylic acid) (PAA) as the polyacid and either poly $(\mathrm{N}, \mathrm{N}-$ dimethylaminoethyl methacrylate, PDMAEMA) or poly(diallyldimethyl ammonium chloride), PDADMAC, as the polybase. ${ }^{7}$ The weight fraction of water varied from 0.4 to 0.8 in these systems. In the mixture PAA-PDMAEMA, Spruijt et al. ${ }^{8}$ measured water fractions $>0.7$ which increased with salt concentration. Another special feature of the coacervate phase is that it displays a much higher viscosity compared to the initial PEL solutions. Liu and co-workers reported an increase by three orders of magnitude in the viscosity of the coacervate phase formed between PAA and PDADMAC compared to the individual PEL solutions. ${ }^{4}$ Finally, low interfacial tensions $\left(\leq 1 \mathrm{mN} \mathrm{m}^{-1}\right)$ between the coacervate phase and the coexisting supernatant phase are common. ${ }^{9-11}$

One of the most important applications of complex coacervation is in microencapsulation. ${ }^{5}$ In this process, a substance (also called core material) which can be liquid or solid in nature, is encapsulated within a layer of coacervate phase. ${ }^{12}$ Thus, oil drops or solid particles in water may be coated with this phase. For the encapsulation to be successful the adsorption of the complex onto the core material and its wettability are key parameters to consider. ${ }^{13}$ The complex coacervate has to spread spontaneously over the surface of dispersed liquid droplets or particles and coat them to form a capsule. ${ }^{14}$ This ability can be assessed by consideration of the relevant spreading coefficients, for which positive values indicate spontaneous spreading. By determining the interfacial tensions between different pairs of the three phases (core, supernatant and coacervate), three spreading coefficients $(S)$ can be defined

$$
\begin{aligned}
& S_{1}=\gamma_{23}-\left(\gamma_{12}+\gamma_{13}\right) \\
& S_{2}=\gamma_{13}-\left(\gamma_{12}+\gamma_{23}\right) \\
& S_{3}=\gamma_{12}-\left(\gamma_{13}+\gamma_{23}\right)
\end{aligned}
$$

where the subscripts 1, 2 and 3 refer to the core material (here oil), the aqueous phase and the coacervate, respectively. Thus, $S_{1}$ is the spreading coefficient for oil at the water-coacervate interface etc. If we assume that $\gamma_{23}$ (water-coacervate) $<\gamma_{12}$ (oil-water) as mentioned above, then $S_{1}$ is always negative and only three possible combinations of the spreading coefficients arise depending on the relative magnitudes of $\mathrm{S}_{2}$ and $\mathrm{S}_{3}:{ }^{15}$

$$
S_{1}<0 ; S_{2}<0 ; S_{3}>0 \text { complete engulfing }
$$




$$
\begin{aligned}
& S_{1}<0 ; S_{2}<0 ; S_{3}<0 \text { partial engulfing } \\
& S_{1}<0 ; S_{2}>0 ; S_{3}<0 \text { non-engulfing }
\end{aligned}
$$

A microcapsule containing a liquid core is similar to an emulsion droplet. As emulsions are thermodynamically unstable, a surface-active material or emulsifier should be added to protect the formed drops from coalescence. ${ }^{16,17}$ In the past, both emulsions and microcapsules stabilised by protein (anionic)-polysaccharide (cationic) complexes have been extensively reported. Two different preparations are described. ${ }^{18}$ The first consists of the addition of oil to an aqueous solution containing the protein-polysaccharide complexes followed by homogenisation. The second involves the formation first of a primary emulsion stabilised by protein, followed by the addition of the polysaccharide which adsorbs onto the protein layer forming a bilayer/multilayer. Jourdain et al. ${ }^{19}$ found an improvement in emulsion stability by using the complexes prepared in water instead of adding the protein and the polysaccharide in two homogenisation steps. Bridging flocculation occurred with the second method while discrete dispersed oil droplets were achieved by using the complexes formed before homogenisation. They related these differences to the structure of the composite biopolymer at the interface that varies depending on the method employed. In all cases studied, the complex at the emulsion droplet surface enhances emulsion stability compared with emulsions of protein alone.

Despite the numerous examples of emulsions stabilised by protein-polysaccharide mixtures encountered in the literature, ${ }^{19-21}$ in all cases the protein acts as a good emulsifier alone, i.e. is surface-active, and in some cases so does the polysaccharide. In this work we extend the idea introduced in our previous paper in which we investigated whether PEC formed between two oppositely charged strong polyelectrolytes (polystyrene sodium sulfonate, PSSNa and PDADMAC) were capable of stabilising oil-water emulsions. ${ }^{22}$ The novelty of this system compared to those containing protein-polysaccharide complexes is that neither polyelectrolyte is surface-active alone and emulsion stabilisation was shown to be due to the adsorption of solid particles of PEC pre-formed in water on the surface of oil drops. Here, we study emulsion stabilisation in a different system of water-soluble polymers constituted by a strong (PDADMAC) and a weak (PAANa) polyelectrolyte in an attempt to establish the general pattern of behaviour in both aqueous PEC dispersions and emulsions containing them. For this system, unlike the first one, ${ }^{22}$ the effect of $\mathrm{pH}$ is an important consideration as PAANa will be uncharged at low $\mathrm{pH}$ and fully charged at high $\mathrm{pH}$. As a result, this will have an impact on the 
strength of the interaction between oppositely charged polymers; we know of no other study reporting the behaviour over a wide range of $\mathrm{pH}$. We first study the behaviour of aqueous mixtures of the two polymers at different $\mathrm{pH}$ and mole fraction of each polyelectrolyte. The size and charge of the obtained complexes are measured and the type of associative phase separation is carefully appraised. Both solid precipitates and coacervate droplets form along the studied $\mathrm{pH}$ range (2-10), while for the case of two strong PELs only precipitates were detected. ${ }^{22}$ Moreover, despite expecting this system at high $\mathrm{pH}$ to behave like that of two strong PELs, coacervate droplets are preferred instead of precipitates. Emulsions stabilised by coacervate droplets are then prepared from the aqueous polymer mixtures at $\mathrm{pH}=10$ and oil and their stability and the arrangement of the complexes around drops is evaluated. Emulsions prepared with PEC precipitates are not stable however probably due to their large size, their relatively low amount or their inherent hydrophilicity. Finally, a method to determine the surface energy of the coacervate phase is described. To conclude, calculations of the three spreading coefficients for systems containing water, coacervate and various oils requiring measurement of the interfacial tension between the coacervate phase and water are given to predict the equilibrium configuration between the three phases. These are compared with the configuration established by experiment.

\section{EXPERIMENTAL}

\subsection{Materials}

Poly(acrylic acid) sodium salt, PAANa, and poly(diallyldimethylammonium chloride), PDADMAC were purchased from Polymer Standard Services (PSS, Mainz) and were used as received. Their chemical structures as well as some other properties are shown in Table 1. The two samples were chosen to have a similar average molecular weight (Mw) and a relatively low polydispersity index. For emulsions, five different oils were selected (Table 1). They include non-polar alkanes, an aromatic oil and a silicone oil. Prior to use, all the oils were passed twice through a basic alumina column (particle size: $0.063-0.200 \mathrm{~mm}$, Merck kGaA) to remove polar impurities. Water was first passed through a reverse osmosis unit and then a Milli-Q reagent water system (Millipore). After treatment, its surface tension measured with a Krüss K11 tensiometer and Wilhelmy plate was $72.0 \mathrm{mN} \mathrm{m}^{-1}$ at $25^{\circ} \mathrm{C}$. Hydrochloric acid, $\mathrm{HCl}$ (Fisher Chemical, 37\%) and sodium hydroxide, $\mathrm{NaOH}$ (Fisher Scientific, >97\%) were used as received. Four liquids and water were selected to estimate the surface energy of the dry coacervate phase from contact angle measurements. They include glycerol (VWR Chemicals, 
98\%), formamide (> 99\%), $\alpha$-bromonaphthalene (97\%) and $n$-hexadecane (99\%). The latter three were purchased from Sigma-Aldrich.

\subsection{Methods}

(a) Preparation and characterisation of aqueous PEC dispersions

A potentiometric titration for PAANa was performed with a calibrated $\mathrm{pH}$ meter $(3510$, Jenway) to determine the degree of ionisation as a function of $\mathrm{pH}$. The natural $\mathrm{pH}$ of a $1 \mathrm{~g} \mathrm{~L}^{-1}$ PAANa solution was 9.7. The $\mathrm{pH}$ was increased to 12 with $\mathrm{NaOH}$ and the solution was titrated with $0.1 \mathrm{M} \mathrm{HCl}$. Individual PEL solutions of different concentrations $(0.01,5,10$ and $30 \mathrm{~g} \mathrm{~L}$ $\left.{ }^{1}\right)$ were prepared by weighing the corresponding amount of each PEL and dissolving it in Milli$\mathrm{Q}$ water. PEL solutions were adjusted to the desired $\mathrm{pH}$ with $\mathrm{NaOH}$ and $\mathrm{HCl}$ solutions of various concentrations. Aqueous PEC dispersions of different mole fractions of PAANa (XPAANa) were obtained by mixing known volumes of each individual PEL of a fixed concentration and $\mathrm{pH}$ with a magnetic stirrer (VWR VMS-C7, stirrer speed $=3$ ) at room temperature. We quote the initial $\mathrm{pH}$ before mixing in all cases. All solutions were prepared in $14 \mathrm{~mL}$ screw-cap glass vials. Due to the influence of the mixing procedure on the characteristics of the resulting PEC structures, we decided to work under fixed conditions in order to obtain reproducible results. The protocol detailed in the previous paper was followed. ${ }^{22}$ The order of addition was set as: water, PAANa solution, PDADMAC solution. Water and PAANa solution were added in one shot additions with a micropipette. PDADMAC solution was then added to the above mixture whilst stirring. The addition of PDADMAC was done sequentially every minute up to the total desired volume with a micropipette. This was done to allow the added polyelectrolyte to interact with the oppositely charged species present in the vial. Therefore, as an example, for the dispersion with XPAANa $=0.1$, each PDADMAC addition was of $1000 \mu \mathrm{L}$ while for the dispersion with $\mathrm{X}_{\mathrm{PAANa}}=0.9$ each addition was of $125 \mu \mathrm{L}$. With this protocol, the total mixing time for all the samples was around $3 \mathrm{~min}$. After the complete addition of PDADMAC solution, mixing was kept at the same speed for an additional minute.

Dynamic light scattering (DLS) employing the cumulant method ${ }^{23}$ was used to determine the average diameter of the complex present in the aqueous dispersions prepared at different XPAANa and $\mathrm{pH}$ with no added electrolyte. Measurements were carried out at $25^{\circ} \mathrm{C}$ using a Zetasizer Nanoseries NanoZS (ZEN3600, Malvern Instruments) and samples were placed in a plastic disposal cuvette of $1 \mathrm{~cm}$ path length. The instrument was equipped with a $4 \mathrm{~mW}$ He-Ne laser beam as a light source, operating at $\lambda=633 \mathrm{~nm}$ under a scattering angle of 
$173^{\circ}$. The results are given as the average of three measurements. The zeta potential was measured at $25{ }^{\circ} \mathrm{C}$ by the same instrument. Measurements were made by introducing a universal dip cell (ZEN1002, Malvern Instruments) inside a plastic disposal cuvette. The Smoluchowski approximation ${ }^{24}$ was used to convert measured electrophoretic mobilities to zeta potentials. Each value was averaged from three parallel measurements. The refractive indices of water and the coacervate phase were obtained using a refractometer (M46 313, Hilger) and a sodium lamp $(\lambda=589 \mathrm{~nm})$ at $25^{\circ} \mathrm{C}$ and were 1.333 and 1.395 , respectively. The refractive index of the coacervate phase was measured in triplicate from the viscous phase obtained after mixing equimolar concentrations of each PEL solution $\left(30 \mathrm{~g} \mathrm{~L}^{-1}\right)$ at $\mathrm{pH}=10$.

In order to characterise the type of associative phase separation (precipitation or complex coacervation) achieved at different $\mathrm{pH}$, visual inspection of the vials together with optical microscope imaging was carried out. Micrographs of a drop of the aqueous PEC dispersion were obtained on a glass slide (Fisher Scientific) using an Olympus BX-51 microscope fitted with a DP50 digital camera. The occurrence of both the coacervate phase and the precipitate was further investigated by centrifugation of $1 \mathrm{~mL}$ of the aqueous PEC dispersion with a minicentrifuge (Minispin plus, Eppendorf) at 10,000 rpm for different periods of time. Optical microscope images of the different phases separated were also taken.

The determination of the water content of the coacervate phase was performed following the procedure reported in refs. 7 and 8. The aqueous PEC dispersion in this case was prepared from the $30 \mathrm{~g} \mathrm{~L}^{-1}$ PEL solutions at $\mathrm{pH}=10(\mathrm{XPAANa}=0.5)$. The coacervate phase was placed on a clean glass slide and water was allowed to evaporate in an oven at $100{ }^{\circ} \mathrm{C}$ until constant weight. The water content is given as the average of three measurements.

Transmission electron microscopy (TEM) images were taken of an aqueous PEC dispersion $\left([\mathrm{PEL}]=5 \mathrm{~g} \mathrm{~L}^{-1}, \mathrm{XPAANa}=0.5, \mathrm{pH}=10\right)$ which forms coacervate droplets. Two experimental procedures regarding the sample preparation were carried out. For the unstained case, $5 \mu \mathrm{L}$ of the aqueous PEC dispersion were placed on a carbon coated copper grid and the sample was air dried. For the stained case, a drop of the sample was placed on parafilm. The grid with the carbon film facing down was placed onto the drop for $2 \mathrm{~min}$. Afterwards, the grid was placed in contact with a drop of Milli-Q water at $\mathrm{pH}=10$. Then, the grid was left for 1 min in a $1 \%$ uranyl acetate solution. The excess of liquid was removed with filter paper after each step. Finally the sample was air dried. TEM images were taken with a JEOL 2010 Transmission Electron Microscope equipped with a Gatan Ultrascan 4000 camera at a voltage 
of $120 \mathrm{kV}$. Energy dispersive X-ray Microanalysis (EDX, Oxford Instruments) using INCA Energy software was also conducted.

\section{(b) Preparation and characterisation of emulsions}

Emulsions composed of an aqueous PEC dispersion and oil were prepared in $14 \mathrm{~mL}$ screwcap glass vials. The two phases were emulsified with an Ultra-Turrax homogenizer (IKA® T25 digital) having a dispersing element of $8 \mathrm{~mm}$ (stator diameter). Mixing was maintained for 2 min at a constant speed of $13,000 \mathrm{rpm}$. Different sets of emulsions were systematically prepared by varying one of the following parameters: concentration of the starting PEL solutions, $\mathrm{X}_{\mathrm{PAANa}}, \mathrm{pH}$ of the initial PEL solutions and oil type. Photos of the vessels were taken just after preparation and at subsequent times to evaluate the emulsion stability. Micrographs of the freshly prepared emulsions were obtained on a dimple glass slide (Fisher Scientific) using an Olympus BX-51 microscope fitted with a DP50 digital camera. The mean droplet diameter of the emulsion was calculated from at least fifty individual droplets on digital micrographs with Image J $1.47 \mathrm{v}$. The amount of oil released from the emulsion after some time was measured by weight, after removing it carefully from above the emulsion with a Pasteur pipette.

A selected emulsion was imaged with cryo-Scanning Electron Microscopy (cryo-SEM). A small volume of emulsion was mounted on an aluminium sample holder (diameter $\sim 10 \mathrm{~mm}$ ) with a spatula. The sample was plunged into liquid nitrogen, previously turned into a slush to decrease the temperature to $-210{ }^{\circ} \mathrm{C}$. The frozen sample was placed inside the cryo-preparation chamber (PP3010T, Quorum Technologies Ltd.) where it was fractured with a sharp knife at $140{ }^{\circ} \mathrm{C}$ under high vacuum. Sublimation of the surface water (ice) was performed inside the Zeiss EVO $60 \mathrm{SEM}$ chamber at $-75^{\circ} \mathrm{C}$ for $10 \mathrm{~min}$ to obtain a clearer image of the droplet interface. Afterwards, the sample was coated with platinum to a thickness of $\sim 2 \mathrm{~nm}$ in the preparation chamber. Finally, it was transferred back to the SEM chamber for imaging at a voltage of $15 \mathrm{kV}$ and a probe current of $30 \mathrm{pA}$.

\section{(c) Determination of spreading coefficients}

The three spreading coefficients for the system oil-water-coacervate were calculated from the measured interfacial tensions between the different phases $\left(\gamma_{12}, \gamma_{23}\right.$ and $\left.\gamma_{13}\right)$. The oils under study are those included in Table 1 . The interfacial tension between oil and water $\left(\gamma_{12}\right)$ was measured with a Krüss K11 tensiometer and the du Noüy ring method at $25^{\circ} \mathrm{C}$. The radius 
of the Pt-Ir ring was $9.545 \mathrm{~mm}$ and the wire diameter was $0.37 \mathrm{~mm}$. The applied correction factors were taken from Harkins and Jordan. ${ }^{25}$ The results of three separate measurements were averaged. After each measurement, the ring was rinsed with ethanol and heated to glowing in a blue Bunsen flame. The two remaining interfacial tensions $\left(\gamma_{23}\right.$ and $\left.\gamma_{13}\right)$ were calculated from the appropriate Young's equation (equations 7 and 8). For convenience the phases 1 (oil), 2 (water) and 3 (coacervate) have been re-named as $o, w$ and $c$, respectively. Therefore, the two interfacial tensions to calculate are designated as $\gamma_{w c}$ and $\gamma_{o c}$.

$$
\begin{aligned}
& \gamma_{a c}=\gamma_{w c}+\gamma_{a w} \cos \theta_{a w}(\text { water drop in air on coacervate) } \\
& \gamma_{a c}=\gamma_{o c}+\gamma_{a o} \cos \theta_{a o}(\text { oil drop in air on coacervate) }
\end{aligned}
$$

where $a$ signifies air. The surface energy of the coacervate $\left(\gamma_{a c}\right)$ was determined by using an indirect method in which the surface energy of a material is split into a polar $\left(\gamma^{p}\right)$ and a nonpolar component $\left(\gamma^{d}\right)$. As described earlier, ${ }^{26,27}$ the contact angle $\theta_{a l}$ of a liquid $(l)$ drop in air on a perfectly smooth and homogeneous surface (here $c$ ) can be written in terms of the polar and dispersion components of the surface energies of the liquid and solid as stated in equation 9 ,

$$
\frac{1}{2} \gamma_{a l}\left(1+\cos \theta_{a l}\right)=\sqrt{\gamma_{a l}^{d} \gamma_{a c}^{d}}+\sqrt{\gamma_{a l}^{p} \gamma_{a c}^{p}}
$$

The two unknowns in this equation are $\gamma_{a c}^{d}$ and $\gamma_{a c}^{p}$ which can be determined by solving two of the above equations simultaneously with the values for two different liquids. However, it is advisable to use more than two liquids of different polarity. For this purpose, water, glycerol, formamide, $\alpha$-bromonaphthalene and n-hexadecane were used. The values of $\theta_{a l}$ of the five probe liquids were measured with a Krüss DSA Mk 10 apparatus with the static sessile drop method, by obtaining the profile of a liquid droplet on a coated glass slide with the coacervate phase. In order to do so, the coacervate phase obtained after mixing $30 \mathrm{~g} \mathrm{~L}^{-1}$ individual PEL solutions at $\mathrm{pH}=10$ was spread carefully on a clean glass slide and water was allowed to evaporate completely in an oven at $100{ }^{\circ} \mathrm{C}$. After that, between 4 and $10 \mu \mathrm{L}$ of the test liquids were placed on the slide with a needle. The circle method was used to measure contact angles below $20^{\circ}$ while the Young-Laplace fitting was applied for contact angles above $20^{\circ}$. When the contact angle between the liquid and the coacervate coated surface was too low to be measured accurately, it was taken to be $<5^{\circ}$. The contact angle values are given as the average of three independent measurements. 
The least squares calculation was carried out to determine the best combination of $\gamma_{a c}^{d}$ and $\gamma_{a c}^{p}$ that fits all the data simultaneously. A 3-D surface energy diagram is obtained, which represents the goodness of fit (inverse of the sum of the squares of variances) to contact angle, set against a matrix of possible values of $\gamma_{a c}^{d}$ and $\gamma_{a c}^{p}$. The values that best fit all the contact angles are read from the coordinates that define the peak. Finally, the surface tensions $\left(\gamma_{a w}\right.$ and $\left.\gamma_{a o}\right)$ and the contact angles $\left(\theta_{a w}\right.$ and $\left.\theta_{a o}\right)$ in equations 7 and 8 are measured with the du Noüy ring method and the Krüss DSA instrument, respectively. The procedure followed for these measurements is the same as stated above. This allows us to calculate the two remaining interfacial tensions in equations 7 and $8\left(\gamma_{w c}\right.$ and $\left.\gamma_{o c}\right)$ and hence the three $\mathrm{S}$ values defined in equations 1-3.

\section{RESULTS AND DISCUSSION}

\subsection{Characterisation of aqueous PEC dispersions}

Aqueous PEC dispersions and oil-water emulsions were studied in detail for mixtures of PDADMAC and PAANa. Since PDADMAC is a strong polybase, it is fully ionised at all $\mathrm{pH}$. On the contrary, PAANa is a weak polyacid. Therefore, its degree of ionisation varies with $\mathrm{pH}$. A potentiometric titration of a $1 \mathrm{~g} \mathrm{~L}^{-1}$ PAANa solution was carried out in triplicate to determine the degree of ionisation as a function of $\mathrm{pH}$ and consequently the $\mathrm{pK}_{\mathrm{a}}$ of the polyacid (Figure S1). The $\mathrm{pK}_{\mathrm{a}}$ value for our sample of PAANa was found to be 6.6 , in reasonable agreement with the literature whose values range from 5.5 to $6.8 .^{28,29}$ It is worth noting the increased $\mathrm{pK}_{\mathrm{a}}$ of a polyacid compared to the value of a low molecular weight analogue $\left(\mathrm{pK}_{\mathrm{a}}=\right.$ 4.2). The behaviour of weak polyelectrolytes upon increasing or decreasing the charge density is not equivalent to the case of low molecular weight electrolytes. In a polyelectrolyte, not all the counterions will dissociate from the polymer chain. In fact, as the magnitude of the charge on the chain increases (by dissociation), it becomes progressively more difficult to remove the next proton due to the close proximity of charged groups. When the polyelectrolyte is strongly charged, some of the counterions remain bound to the polymer chain due to the large electrostatic potential on the chain, thereby reducing the effective charge of the polyion. This is known as counterion condensation and is described by Manning's theory. ${ }^{30}$

(a) Low [PEL] 
As mentioned above, the $\mathrm{pH}$ is a key parameter to consider in the study of PEC formation in this polyelectrolyte mixture. Aqueous PEC dispersions at low concentration were first characterised in terms of their average diameter and zeta potential. The appearance of aqueous PEC dispersions prepared from $0.1 \mathrm{~g} \mathrm{~L}^{-1}$ PEL solutions at different XPAANa and $\mathrm{pH}$ is shown in Figure 1. Here, $\mathrm{x}$ refers to the mole fraction using the values of Mw given in Table 1 (131.2 kDa for PAANa and $174 \mathrm{kDa}$ for PDADMAC). In general, all aqueous PEC dispersions and solutions of the neat polymers are transparent and colourless. However, some mixtures at specific XPAANa are bluish or show signs of precipitation. Although Petrov et al. ${ }^{31}$ and Vitorazi et al. ${ }^{32}$ measure the reduction in $\mathrm{pH}$ after mixing due to proton release around the $\mathrm{pK}_{\mathrm{a}}$, we note that the majority of work in this area makes no mention of this. ${ }^{4,73}$ The average diameter of the entities obtained after mixing the polyelectrolyte solutions at selected $\mathrm{pH}$ was measured and are shown in Figure S2. Despite the occasional high values (which match with samples that are more hazy), there is a tendency for the average diameter of the complex to decrease upon increasing XPAANa from around $120 \mathrm{~nm}$ to around $60 \mathrm{~nm}$. At $\mathrm{pH}=2$ (not shown), despite not expecting complexes to be formed through electrostatic interactions as PAANa is fully protonated, monomodal distributions centred around $100 \mathrm{~nm}$ at XPAANa $<0.64$ were present. At $\mathrm{pH}=12$ one would expect to obtain complexes through electrostatic interactions as both polyelectrolytes are fully charged. Monomodal distributions were only obtained however for the samples prepared with $\mathrm{X}_{\mathrm{PAANa}}=0.34$ and 0.55 (diameters $382 \mathrm{~nm}$ and $74 \mathrm{~nm}$, respectively). At the other mole fractions, the samples were too polydisperse for cumulant analysis and in some cases the size distribution showed more than one peak. Therefore, from the results obtained at extreme $\mathrm{pH}$ values, one can state that the interactions in the system PDADMAC + PAANa cannot be ruled by electrostatics alone. This associative phase separation has to be also mediated through additional inter-molecular interactions, such as van der Waals, hydrogen bonding or hydrophobic and dipole interactions. ${ }^{34}$ This agrees with previous work from Alonso et al. ${ }^{35}$ They detected aggregates at $\mathrm{pH}=3$ where PAA is not ionised. Their occurrence was explained by the formation of H-bonds between different chains of PAA. ${ }^{35}$ The carboxylic acid moiety is a highly polar functional group due to the strongly polarized carbonyl and hydroxyl groups which can interact through hydrogen bonding with other carboxylic acids or water molecules. ${ }^{35}$ At low $\mathrm{pH}$, the authors also pointed out the possibility that PDADMAC could form complexes with single chains or small aggregates of PAA. At high $\mathrm{pH}$, no complex was detected at $\mathrm{pH}=13$. By increasing the $\mathrm{pH}$, the charge density of PAA increases. As a result, there is an increase in the amount of water linked to the polymer through hydrogen bonding. ${ }^{35}$ Conversely, the charges in PDADMAC are given by a quaternary amine surrounded by an 
organic environment, which makes it less favourable for structured water. ${ }^{35}$ At intermediate $\mathrm{pH}$ these quaternary amines can destroy the water shell that surrounds the carboxylic acid groups of PAA and electrostatic interaction takes place. ${ }^{35}$ However, with increasing $\mathrm{pH}$, the number of water molecules associated with the carboxylate group increases and the breaking of the water shell by the quaternary amines becomes less likely. ${ }^{35}$ As a result, no complexation is achieved. The authors proved the hindering effect of water molecules since, after the addition of a hydrogen bonding breaker, the complexation at high $\mathrm{pH}$ took place. ${ }^{35}$ However, it is worth mentioning that other parameters such as the chain rigidity and the ionic strength can also affect the strength of the interaction between charged polymers. ${ }^{3,4,36}$

The zeta potential of each mixture at various $\mathrm{pH}$ was also measured (Figure 2). At $\mathrm{pH}$ $=2$ all the dispersions exhibited a positive value of zeta potential. As PAANa is fully protonated, the positive charge is given by the quaternary nitrogen groups in PDADMAC. For the other $\mathrm{pH}$ values, the curve is sigmoidal in shape in which the zeta potential changes from positive values to negative ones on increasing XPAANa. We see that the value of XPAANa at sign reversal is lower at higher $\mathrm{pH}$ (Figure 3). At low $\mathrm{pH}$ values, only a small fraction of PAANa groups is ionised. Therefore, a higher fraction of PAANa chains are required to fully neutralise the PDADMAC charges. As the $\mathrm{pH}$ increases, the change in the sign of the zeta potential occurs at lower XPAANa. From a $\mathrm{pH}$ equal to the $\mathrm{pK}_{\mathrm{a}}$ onwards, the $\mathrm{X}_{\mathrm{PAANa}}$ of zero zeta potential reaches a plateau as expected. Vitorazi et al $^{32}$ performed an extensive study of the same PEL system by using a combination of titration calorimetry, light scattering and electrophoresis. They studied the influence of [PEL], $\mathrm{pH}$ (7 and 10), molecular weight and mixing protocol (direct or stepwise). Our results regarding the size and charge of the obtained entities are in line with their findings. Dispersions of higher turbidity occurred around charge neutrality and the charge of the complexes is governed by the polyelectrolyte in excess. As in ref. 32, we define the charge ratio $\mathrm{Z}_{-/+}$as the ratio of the molar concentration of negative and positive charges (using $\mathrm{Mw}$ of the monomer). The variation of the size of PEC and their zeta potential with $\mathrm{Z}_{-/+}$is plotted in Figure S3 for three extents of ionisation of PAANa. Although the trends are the same, the data does not collapse onto a single curve and we prefer to use XPAANa.

\section{(b) High [PEL]}

In terms of visual inspection at this low concentration, this system is comparable to the system containing PDADMAC + PSSNa (two strong polyelectrolytes) reported previously. ${ }^{22}$ However, things change upon increasing the concentration of the initial PEL solutions. This 
study was completed at four different $\mathrm{pH}$ values covering the entire range, i.e. $\mathrm{pH}=2,4,6$ and 10. Figure 4 shows the appearance of the dispersions prepared at $\mathrm{pH}=4$ and 10 at different XPAANa from PEL solutions at $5 \mathrm{~g} \mathrm{~L}^{-1}$. At $\mathrm{pH}=4$ precipitation in mixtures is observed at all values of XPAANa (i.e. particles form) while at $\mathrm{pH}=10$ no complex precipitated from solution despite the fact that the dispersions were whitish at low and intermediate values of XPAANa. A close inspection of a drop of the dispersion at $\mathrm{pH}=10$ using optical microscopy reveals the presence of coacervate droplets several microns in size in contrast to the solid particles formed at $\mathrm{pH}=4$ (Figure 4(c) and (d), respectively). Both kinds of dispersed phase can be distinguished quite easily as the coacervate droplets are fluid spherical entities whereas the particles are less spherical and solid in nature. The appearance of aqueous PEC dispersions at $\mathrm{pH}=2$ and 6 are included in Figure S4 together with selected optical microscope images of the resulting mixtures. At $\mathrm{pH}=2$ all the dispersions were transparent, apart from the one at XPAANa $=0.92$ which was slightly bluish. At $\mathrm{pH}=6$ all the dispersions were turbid with that of highest turbidity around charge neutralisation. Coacervate droplets were observed in all the mixtures at these two $\mathrm{pH}$ values. Therefore the transitions coacervate - precipitate - coacervate can be observed by increasing the $\mathrm{pH}$. A transition from precipitate to coacervate by increasing the $\mathrm{pH}$ from 4 to 7 was also reported by Jha and co-workers for the same polyelectrolyte system. ${ }^{7}$ However, in the literature regarding the characterisation of PEC in aqueous media, apart from ref. 7 no systematic study describing the type of associative phase separation across all the $\mathrm{pH}$ range exists. Instead, investigations have mainly been focused at a specific $\mathrm{pH}^{4}$ or at two $\mathrm{pH}$ values. $^{32}$

By increasing the concentration of PEL, several different scenarios can occur. The number of complexes could increase at constant size or complexes of larger size may form at constant number or a combination of both could ensue. We have evidence that the size of the PEC (particles or coacervate droplets) increases with PEL concentration at all $\mathrm{pH}$ values. From light scattering measurements at a $[\mathrm{PEL}]=0.1 \mathrm{~g} \mathrm{~L}^{-1}$ the entities had a diameter centred on 100 $\mathrm{nm}$. However, as seen from the optical microscope images in Figure 4 at this higher [PEL], the size of some of the complexes are in the micron range. As gleaned from the results of Starchenko et al. for the system PDADMAC + PSSNa, ${ }^{37}$ upon increasing the PEL concentration the aggregation of primary particles is accelerated and this leads to an increase in the size of secondary particles.

In order to further evaluate the difference between these two types of associative phase separation (precipitation and coacervation) centrifugation of $1 \mathrm{~mL}$ of the aqueous PEC 
dispersions (XPAANa $=0.5$ ) prepared at two different $\mathrm{pH}$ was carried out. The initial concentrations of the PEL solutions used were $5 \mathrm{~g} \mathrm{~L}^{-1}$ and $10 \mathrm{~g} \mathrm{~L}^{-1}$ for $\mathrm{pH}=4$ and 10 , respectively. At $\mathrm{pH}=4$, after centrifugation a white precipitate is collected on the bottom of the Eppendorf tube as seen in Figure 5(2), consistent with the solid-liquid type of phase separation. The appearance of the aqueous PEC dispersion at $\mathrm{pH}=10$ is shown before and after centrifugation in Figure 5(1). The dispersion was initially whitish which after centrifugation yields a phase separated system consistent with the liquid-liquid type of phase separation of the complex coacervate. Three different phases were identified and designated supernatant (phase A), interface (phase B) and coacervate phase (phase C). The supernatant was mostly water depleted in coacervate droplets (Figure 5(A)). Some spherical entities were still evident around the edges of the drop as soon as the water began to evaporate. However, they were hardly visible at the outset. The interface consisted of an aqueous phase rich in coacervate droplets (Figure 4(B)). Images of the same drop placed on a glass slide were taken at different times. Coalescence of the coacervate droplets occurred as after 17 min a substantial increase in their size was detected. After 22 min droplets were no longer visible as they had coalesced to a bulk film of coacervate phase. Finally, the coacervate phase after centrifugation was a transparent and viscous phase concentrated in both polymers (Figure 5(C)). The water content was $63 \pm 3 \%$ which is close $(\sim 66 \%)$ to the value reported for the same system at $\mathrm{pH}=7$. $^{7}$

The entire $\mathrm{pH}$ range was studied at a high PEL concentration $\left(5 \mathrm{~g} \mathrm{~L}^{-1}\right)$ for a mixture containing an equal mole fraction of both polymers (XPAANa $=0.5$ ). Figure $6(\mathrm{a})$ shows the appearance of the vials at different $\mathrm{pH}$ values immediately after preparation and at three subsequent times. The same conclusions regarding the type of associative phase separation arise from this set of experiments as derived from the experiments above. At $\mathrm{pH}=2$ the sample displays turbidity and coacervate droplets are detected despite the possibility that no electrostatic interaction is expected to occur (Figure 6(b)). At pH 3 and 4 both coacervate droplets and solid precipitates were formed (Figure 6(c and d)). The size of the solid or liquid particles range between a few microns and several hundred microns as they aggregate quite easily. Co-appearance of both types of phase separation has been reported in the literature for the system under study. Koetz and Kosmella found co-existence of coacervation and particle flocculation at specific polymer Mw's and PEL concentrations. ${ }^{38}$ From $\mathrm{pH}=5$ onwards, no precipitates are observed either visually or via optical microscopy and only coacervate droplets form. As pointed out in the introduction, it is generally assumed that precipitation rather than coacervation is obtained for strongly interacting polymer pairs. Therefore, one would expect 
precipitate formation at high $\mathrm{pH}$ values which is not the case. This may be related to the enhanced ability of PAANa to form hydrogen bonds at high $\mathrm{pH}$, as explained earlier. Due to the dipoles present in the carboxylic acid moieties, water is associated to them through hydrogen bonding leading to partial screening of the negative charge of the ionic groups. ${ }^{35}$ Therefore, the electrostatic interaction with PDADMAC is weakened and this results in the formation of a complex coacervate containing water instead of a precipitate. The volume fraction of coacervate droplets is higher at $\mathrm{pH} 5$ and $6 c f$. higher $\mathrm{pH}$ and these samples display maximum turbidity initially (Figure 6). One year after preparation the coacervate droplets in these dispersions fully coalesced forming a transparent coacervate phase on the bottom leaving a transparent supernatant solution where few coacervate droplets were still detected. Despite a low interfacial tension between the coacervate droplets and water, ${ }^{9-11}$ since the droplets are very small the Laplace pressure is high which is consistent with the occurrence of coalescence at $\mathrm{pH}=5$ and 6 . From $\mathrm{pH}=7$ to 10 , a white dispersion concentrated in coacervate droplets sediments slowly but complete coalescence is not achieved. Moreover, it easily re-disperses recovering the white dispersion obtained after preparation. Therefore the stability to coalescence of the coacervate droplets at high $\mathrm{pH}$ is considerably improved. It may be that the coacervate droplets at high $\mathrm{pH}$ possess excess of charged polymer at their interfaces which act to prevent their coalescence.

Intrigued by the high stability of coacervate droplets at $\mathrm{pH}=10$, we wondered if they were homogenous from their core to the surface or if a distinct surface layer of mixed polymers formed around the core. TEM images of the coacervate droplets obtained from a fresh aqueous PEC dispersion of composition XPAANa $\approx 0.5,[\mathrm{PEL}]=5 \mathrm{~g} \mathrm{~L}^{-1}$ at $\mathrm{pH}=10$ are shown at different magnifications. Figure 7 shows different areas of the unstained sample, whereas Figure S5 corresponds to the sample negatively stained with uranyl acetate. The uranyl acetate crystals surround the structures giving a 3D appearance. In all cases, near spherical droplets ranging in diameter between $200 \mathrm{~nm}$ and $500 \mathrm{~nm}$ are observed, although some are distinctly non-spherical. Since water evaporates from the droplets during preparation, the size of the remaining concentrated polymer droplet will be less than that in the original dispersion (fully hydrated). The droplets are homogeneous in texture and exhibit no internal ordering. For the unstained case, the droplets appear to be surrounded by very small particulates (black dots in Figure 7). For the stained sample, these particulates are not visible as uranyl acetate crystals surround the droplets. In general, the coacervate droplets have the same appearance as those observed with optical microscopy (Figure 4(c)) despite the latter being of larger size. The optical microscope 
image was obtained around $1 \mathrm{hr}$ after sample preparation where it is likely that some droplet coalescence has occurred. Additional TEM images of coacervate droplets are shown in Figure S6. Using EDX analysis, no special element was detected. In fact the polyelectrolytes are composed mainly of $\mathrm{C}$ and in lower proportion $\mathrm{N}, \mathrm{O}, \mathrm{Na}$ and $\mathrm{Cl}$. Since the TEM grid is carbon coated, carbon was one of the main peaks displayed in the spectra (data not shown). The small particulates have not been identified. Using this technique, we conclude that the coacervate droplets are homogeneous and do not appear to possess any internal structure or surface shell. We confirm that their size falls in the nm range and that they are spherical in shape. However, the reason for their coalescence stability at high $\mathrm{pH}$ remains to be established. Few TEM images of the coacervate phase in mixed polymer systems exist in the literature. ${ }^{39,40}$ They also display a homogeneous texture without internal ordering or evidence of a surface shell or membrane.

\subsection{Preparation and characterisation of oil-in-water emulsions}

Our interest here is whether the PEC prepared in water are surface-active enough to adsorb to an oil-water interface created on emulsifying the aqueous phase with the non-polar alkane dodecane. In our earlier work in the system PDADMAC + $\mathrm{PSSNa}^{22}$ the oil-in-water emulsion stability was enhanced by increasing the initial concentration of PEL. Moreover, on varying XPSSNa, the emulsion droplet size and the extent of coalescence were reduced upon approaching the conditions leading to charge neutrality. In that case, the oil was added in one step during homogenisation. As identified in the previous section, for the system PDADMAC + PAANa, $\mathrm{pH}$ has a dramatic influence on the associative phase separation that takes place. Therefore, this parameter will be a key one in the study of emulsion stabilisation. Figure S7 shows the appearance of the emulsions obtained from the aqueous PEC dispersions prepared at $\mathrm{pH}$ values from 2 to 6 from $5 \mathrm{~g} \mathrm{~L}^{-1}$ PEL solutions at XPAANa $=0.5$. At $\mathrm{pH}=2$ (coacervate), an o/w emulsion was formed after mixing which creamed rapidly and completely phase separated within 11 days after preparation. An air-in-water foam was also formed during mixing which was only stable for a few min. This surface activity is most likely connected with the presence of fully protonated poly(acrylic acid). In order to verify this assumption, emulsions containing the individual PEL solutions at the same concentration and $\mathrm{pH}$ were prepared with dodecane. That with PDADMAC coalesced rapidly after preparation until complete phase separation whilst emulsion formation with PAANa produces considerable amount of foam and the emulsion was stable for a longer period of time compared to that formed from the aqueous PEC dispersion. This is consistent with the findings of Ishimuro and Ueberreiter ${ }^{41}$ who showed that the airwater surface tension was lowered at low $\mathrm{pH}$ on adding PAA (uncharged) whereas PAA was 
not surface-active at higher $\mathrm{pH}$ (charged). Further investigation of the ability of the PAANa solution at $\mathrm{pH}=2$ to stabilise emulsions was carried out. Solutions of PAANa at different concentrations ( 0.05 to $5 \mathrm{~g} \mathrm{~L}^{-1}$ ) were prepared and emulsions were obtained following the standard procedure. As seen in Figure S8, o/w emulsions prepared at 0.05 and $0.10 \mathrm{~g} \mathrm{~L}^{-1}$ coalesced completely in less than 10 min after preparation and no air bubbles persisted during high-shear homogenisation. On the other hand, emulsions prepared from solutions at higher concentration (>0.50 $\mathrm{g} \mathrm{L}^{-1}$ ) generated bubbles while mixing and a stable emulsion cream was achieved for a longer period of time despite some coalescence of oil. The concentration of PAANa required to display surface activity therefore must be $>0.10 \mathrm{~g} \mathrm{~L}^{-1}$.

At $\mathrm{pH}$ values of 3 and 4 where coacervate droplets and precipitate particles co-exist in water, no stable emulsions were obtained either (Figure S7). The reasons may be that the number of particles and coacervate drops is not sufficient for stabilisation or that particles/aggregates are too large to remain attached to droplet interfaces or that they are too hydrophilic in nature. In order to increase the overall particle concentration, aqueous PEC dispersions were prepared from the PEL solutions at $10 \mathrm{~g} \mathrm{~L}^{-1}$. However, this was not sufficient as the emulsion completely coalesced after homogenisation (data not shown). At pH 5 and 6 (coacervate), an unexpected situation was encountered. At these $\mathrm{pH}$ aqueous PEC dispersions are the most turbid. However, after homogenisation of oil, the aqueous phase became almost transparent at $\mathrm{pH}=6$ and no emulsion was obtained. It was noticed that the coacervate droplets coalesced on the head of the homogeniser and did not take part in emulsion stabilisation. At $\mathrm{pH}=5$, an initial viscous emulsion collected on the top of the vial; it was not stable for a long period of time however. In this case the turbidity of the separated aqueous solution decreased as well due to the coalescence of some of the coacervate phase on the head of the homogeniser. The highly viscous nature of these particular coacervate systems makes it difficult with respect to emulsification.

For $\mathrm{pH}$ values between 7 and 10 (coacervate), the behaviour was similar so the discussion will be focused on the emulsion prepared at $\mathrm{pH}=10$. Emulsions prepared with the individual PEL were not stable as complete phase separation was obtained after homogenisation (Figure S9). For the PEL mixture, two different preparations were evaluated $\left(\phi_{o}=0.2\right)$ : addition of oil in one step and addition of oil stepwise summarised in Figure 8. For the emulsion prepared with the addition of oil stepwise, the oil was added in aliquots of 200 $\mu \mathrm{L}$ and homogenised with the Ultra-turrax homogeniser each time. Between each addition, the 
emulsion was left to stand for a few hours to assess its stability. In the case of the addition of oil in one step, the emulsion was virtually completely phase separated in less than 1 day. The extent of coalescence was high but there was a residual volume of emulsion cream around the walls of the vial. For the emulsion prepared stepwise, the emulsion cream was stable for a longer period of time. The final emulsion containing $\phi_{o}=0.20$ liberated around $10 \%$ of oil by coalescence but was subsequently stable for at least 6 months (Figure 8(b)). The viscosity of the emulsion increased with an increase in $\phi_{o}$ as expected. Since the average drop diameter was independent of $\phi_{o}$ (between 24 and $28 \mu \mathrm{m}$ ), it implies that the additional oil does not swell existing drops stabilised by coacervate but is used in creating new drops which become stabilised. In order to compare the one step and multistep protocols more correctly, an extra experiment was performed. Oil was added in one step and then five homogenisations were carried out leaving the emulsion to stand for $1 \mathrm{hr}$ between each step. No stable emulsion could be prepared either with this modified procedure. This strongly highlights the importance of the addition of oil stepwise in obtaining a stable emulsion.

Forgiarini et ll $^{42}$ studied the system water/nonionic surfactant/decane to obtain nanoemulsions by three different emulsification methods: (i) stepwise addition of oil to a watersurfactant mixture, (ii) stepwise addition of water to an oil-surfactant mixture and (iii) mixing of all components simultaneously. Although the nature of the emulsifier is different to the one studied here, nanoemulsions with high kinetic stability were only obtained by method (ii). In our case, the reason why a stable emulsion can only be prepared with the addition of oil stepwise is not fully understood. On the one hand, the kinetics of adsorption of the coacervate phase onto the oil-water interface could be relatively slow compared to that of surfactant molecules. Therefore, the subsequent addition of oil followed by homogenisation may enable a more homogeneous coating around oil drops to be formed. In fact it is common during industrial manufacture of emulsions that the dispersed phase (here oil) is drip fed into the reactor during mixing as this allows good dispersion and stabilisation in a more controlled manner.

Optical microscope images were obtained by placing a sample of fresh emulsion on a glass slide without a coverslip (Figure 9(a)). As seen in the left hand image, the central group of droplets are enveloped by a film which is most likely that of coacervate. On the right hand image, concentric spheres are visible and in some cases the inner sphere possesses nodules or horns. These kind of structures were reported in a study of microencapsulation in which oil 
droplets were surrounded by the coacervate complex prepared between a protein and a polysaccharide. ${ }^{43}$ Cryo-SEM images of a selected emulsion (Figure 9(b)) show the curvature of the oil-water interface with the interior of the oil droplet on the left and the adjacent continuous phase on the right. Both the interface and the aqueous phase contain frozen, monodisperse spherical entities which are attributed to coacervate droplets of diameter around $150 \mathrm{~nm}$. The different appearance of the interface around oil drops observed via optical microscopy and SEM is at first sight puzzling. From optical microscope images, what appears to be a continuous film is observed which may indicate that coacervate droplets coalesce during emulsification. It may be however that sub-micron coacervate droplets do not coalesce but adsorb to drop interfaces and cannot be resolved optically. Cryo-SEM images reveal individual spherical entities which are probably frozen coacervate droplets, i.e. implying that intact coacervate droplets aggregate at the oil-water interface in multilayers.

In order to enhance the emulsion stability, an aqueous PEC dispersion prepared from $30 \mathrm{~g} \mathrm{~L}^{-1} \mathrm{PEL}$ solutions at $\mathrm{pH}=10$ was prepared (Figure $\mathrm{S} 10(\mathrm{a})$ ). The dispersion exhibits similar turbidity to the one prepared from $5 \mathrm{~g} \mathrm{~L}^{-1}$ PEL solutions. However, a viscous coacervate phase formed on the bottom of the vial, around the walls and on the magnetic stirrer bar as seen in the inverted vial (Figure S10(d)). Spherical coacervate droplets were visible in the dispersion (Figure S10(c)). However, upon addition of oil and homogenisation, the head of the homogeniser was covered by the viscous coacervate phase and the oil on the top was mixed with it without being emulsified (Figure S10(b)). The aqueous phase after homogenisation was significantly depleted of coacervate droplets (Figure S10(e)). Therefore, systems exhibiting complex coacervation are very sensitive and the stabilisation of emulsions from them has to be optimised for each individual pair of polyelectrolytes.

As the most promising results on emulsion stabilisation were achieved with the aqueous PEC dispersion prepared from $5 \mathrm{~g} \mathrm{~L}^{-1}$ PEL solutions (XPAANa $=0.5$ ) by stepwise addition of oil, we evaluated the emulsion stability in the case of different oils. We observed different behaviour depending on the type of oil. Emulsions of squalane and isopropyl myristate completely phase separate in less than $1 \mathrm{hr}$ after preparation (Figure S11). That of PDMS also coalesces 1 day after preparation but the optical microscope images once creaming had halted reveal the same kind of behaviour as with dodecane: oil droplets surrounded by a coacervate phase (Figure S12). For these three oils, a viscous phase remained at the oil-water interface and around the walls after the majority of oil had coalesced. For toluene, the emulsion was much more stable to coalescence compared with the other oils and optical microscopy reveals a 
different morphology of the oil droplets (Figure S13). Here, the droplets are more deformed and stretched and a large number of them become encased by the coacervate phase.

\subsection{Determination of spreading coefficients}

In microencapsulation, complex coacervates are used to encapsulate hydrophobic core materials (oil drops or solid particles). In order to form core/shell drops (as here), the coacervate phase needs to coat the oil drops resulting in a composite emulsion of oil drops contained within larger coacervate drops. In principle, this coating could occur either by (i) spreading of the coacervate phase at the oil-water interface forming a thin coacervate film with the shell building up by further growth via coalescence of additional coacervate droplets or (ii) individual coacervate droplets surround the oil core without spreading such that the shell is formed by sequential wetting with additional coacervate droplets until a shell is obtained. Two immiscible liquid drops (here oil and coacervate) surrounded by a third immiscible liquid (here water) may form a range of equilibrium configurations depending on the various interfacial tensions $(\gamma)$ and spreading coefficients $(S)$, see equations $1-3 .{ }^{15}$ Based on equilibrium interfacial energy considerations and depending on the sign combinations of the three spreading coefficients, three different scenarios are predicted: complete engulfing, partial engulfing and non-engulfing of the coacervate phase around oil drops in water. At fixed values of $\gamma_{13}$ (oilcoacervate) and $\gamma_{23}$ (water-coacervate), an increase in $\gamma_{12}$ (oil-water) is predicted to promote complete engulfing (from either non- or partial engulfing). For the oils tested in this study we have encountered examples of complete engulfing (dodecane and toluene) and non-engulfing (isopropyl myristate and squalane). However, additional parameters including the viscosity of the coacervate phase affect the kinetics of spreading and, if the shell material possesses shear elasticity, this can actually result in a resistance to spreading. It was further demonstrated that three-phase wetting in coacervate-containing systems depends not only on interfacial energies but on the character of the flow process; a coacervate drop approaching an oil drop in water initially wets and then de-wets the oil-water interface whereas an oil drop in a coacervate phase when released from a needle can penetrate the coacervate-water interface forming a stable compound drop. ${ }^{13}$

In order to evaluate whether our experimental findings are in line with theoretical predictions, $S_{1}, S_{2}$ and $S_{3}$ have been calculated from knowledge of the respective interfacial tensions as explained earlier. For these, $\gamma_{a w}$ was $71.9 \pm 0.2 \mathrm{mN} \mathrm{m}^{-1}$ at $25^{\circ} \mathrm{C}$, in good agreement with the best literature value. ${ }^{44}$ The value of $\gamma_{a o}$ for each oil is shown in Table 2 . The static 
contact angles $\theta_{a w}$ and $\theta_{a o}$ are measured by placing a drop of water or oil in air onto a glass slide coated by the coacervate phase. The film of coacervate phase placed on the glass slide after water removal was completely transparent and homogeneous. For a water drop, $\theta_{a w}$ was $49.6^{\circ}$. All the selected oils appeared by eye to spread completely so $\theta_{a o}$ is considered to be < $5^{\circ}$. In order to determine $\gamma_{a c}^{d}$ and $\gamma_{a c}^{p}$, values of $\gamma_{a l}^{d}$ and $\gamma_{a l}^{p}$ for the different test liquids are given in Table 3 together with the air-liquid contact angles on a glass slide covered with the coacervate phase. The least squares calculation was carried out to determine the best combination of $\gamma_{a c}^{d}$ and $\gamma_{a c}^{p}$ that fits all the data simultaneously. The 3-D surface energy diagram so obtained is shown in Figure 10. The values that best fit all the contact angles are read from the coordinates of the peak. These values are $\gamma_{a c}^{d}=39.3 \mathrm{mN} \mathrm{m}^{-1}$ and $\gamma_{a c}^{p}=11.6 \mathrm{mN}$ $\mathrm{m}^{-1}$ so that $\gamma_{a c}=50.9 \mathrm{mN} \mathrm{m}^{-1}$. In comparison, the surface energy of hydrophilic Crown glass was estimated to be $76 \mathrm{mN} \mathrm{m}^{-1}$, ${ }^{45}$ whereas that for PTFE was estimated to be $18 \mathrm{mN} \mathrm{m}^{-1}$. ${ }^{46}$ Our value for the coacervate phase indicates it is partially hydrophobic originating from its relatively high dispersion component.

By solving equation 7 with the given data, $\gamma_{w c}=4.2 \pm 0.3 \mathrm{mN} \mathrm{m}^{-1}$. This is broadly in agreement with the low values for the interfacial tension of the coacervate phase against the continuous aqueous phase in the literature. However, they tend to be smaller. ${ }^{9-11}$ With our calculated value of $\gamma_{w c}$, the assumption of $\gamma_{w c}<\gamma_{o w}$ stated in the introduction is confirmed. Further, by solving equation 8 for each oil, values for $\gamma_{o c}$ can be calculated (Table 2). They are around $25 \mathrm{mN} \mathrm{m}^{-1}$ and do not change much with oil type. Finally, by substituting the values of $\gamma_{o w}, \gamma_{w c}$ and $\gamma_{o c}$ into equations 1-3, the values of $\mathrm{S}_{1-3}$ can be calculated for each oil and these are also given in Table 2. For dodecane and toluene, the combination of the three spreading coefficients fulfil the condition for complete engulfing observed experimentally. However, for isopropyl myristate and squalane the predicted morphology is complete engulfing although the emulsion was destabilised in less than $2 \mathrm{hr}$. For PDMS, even if the optical microscope images of the freshly prepared emulsion showed oil droplets surrounded by the coacervate phase, the emulsion was broken 1 day after preparation. In these cases the prediction does not reflect the observed morphology. We note that agreement between predicted and observed morphology is not always fulfilled. ${ }^{15,47,48}$ Torza and Mason ${ }^{15}$ found discrepancies in systems when $\mathrm{S}_{2}$ or $\mathrm{S}_{3}$ were quite small (related to the errors in measuring interfacial tensions). Tasker et al. ${ }^{48}$ also noted the discrepancy between the predicted and observed morphologies when the $S_{2}$ values were close to 0 . We accept that our analysis uses as substrate a dried coacervate phase which may possess a surface energy different to that of a hydrated one formed in situ in water. 


\section{CONCLUSIONS}

In aqueous mixtures of a strong (PDADMAC) and a weak (PAANa) polyelectrolyte, both precipitation and coacervation occur as a result of associative phase separation which is dependent on $\mathrm{pH}$. The progression coacervate $\rightarrow$ precipitate/coacervate $\rightarrow$ coacervate occurs upon increasing the $\mathrm{pH}$ as PAANa becomes charged. Although precipitates are expected to be formed when the interactions between the PELs are strong, at high $\mathrm{pH}$ when both polymers are fully ionised complex coacervation yielding droplets in water occurs at all mixing ratios. The weak interaction is explained by the formation of hydrogen bonds between water molecules and carboxylate groups in PAANa that weaken the electrostatic interaction with the quaternary amine groups in PDADMAC. Regarding emulsions prepared from aqueous PEC dispersions, no stable emulsion was possible at low and intermediate $\mathrm{pH}$ where coacervate droplets or coacervate droplets and solid particles coexist exhibiting positive values of the zeta potential. This could be a result of their relatively low amount, their considerable size or their intrinsic hydrophilicity. By contrast, at higher $\mathrm{pH}$ between 7 and 10, stable dodecane-in-water emulsions could be formed from the coacervate phase of near neutral charge if oil is added sequentially. Oil droplets coated by the coacervate phase are observed. The morphology of the oil droplets coated by the coacervate phase is compared with theoretical predictions using equilibrium spreading coefficients for a range of oils. Despite the agreement for dodecane and toluene (complete engulfing), a discrepancy is found for the other oils. It is suggested that kinetic aspects linked to the viscosity of the coacervate phase play a role in the encapsulation process which are not accounted for in equilibrium spreading coefficients. From these results and those in our earlier work, ${ }^{22}$ we are not yet in a position to predict the general pattern of behaviour in both aqueous PEC dispersions and emulsions containing them for mixtures of oppositely charged polymers. Further investigation of other polyelectrolyte combinations is required to develop a better understanding of this area.

\section{ACKNOWLEDGEMENTS}

We are grateful to Shiseido (Japan) for a fully funded PhD studentship to AMBR and to Mr. A. Sinclair and Mrs. A. Lowry (University of Hull) for the SEM and TEM images, respectively. 


\section{REFERENCES}

[1] P.A. Albertsson, Partition of cell particles and macromolecules: distribution and fractionation of cells, mitochondria, chlorophasts, viruses, proteins, nucleic acids, and antigen-antibody complexes in aqueous polymer two-phase systems, Wiley-Interscience, New York, 1971, pp. 18-21.

[2] O. Olabis, L.M. Robeson and M.T. Shaw. Polymer-polymer miscibility, Academic Press, New York, 1979, p. 4.

[3] F. Comert, A.J. Malanowski, F. Azarikia and P.L. Dubin, Coacervation and precipitation in polysaccharide-protein systems, Soft Matter, 2016, 12, 4154-4161.

[4] X. Liu, M. Haddou, I. Grillo, Z. Mana, J-P. Chapel and C. Schatz, Early stage kinetics of polyelectrolyte complex coacervation monitored through stopped-flow light scattering, Soft Matter, 2016, 12, 9030-9038.

[5] H.G. Bungenberg de Jong and H.R. Kruyt, Coacervation (Partial miscibility in colloid systems), Proc. Koninkl. Med. Akad. Wetershap., 1929, 32, 849-856.

[6] E. Kizilay, A.B. Kayitmazer and P.L. Dubin, Complexation and coacervation of polyelectrolytes with oppositely charged colloids, Adv. Colloid Interface Sci., 2011, 167, 2437.

[7] P.K. Jha, P.S. Desai, J. Li and R.G. Larson, pH and salt effects on the associative phase separation of oppositely charged polyelectrolytes, Polymers, 2014, 6, 1414-1436.

[8] E. Spruijt, A.H. Westphal, J.W. Borst, M.A. Cohen Stuart and J. van der Gucht, Binodal compositions of polyelectrolyte complexes, Macromolecules, 2010, 43, 6476-6484.

[9] E. Spruijt, J. Sprakel, M.A. Cohen Stuart and J. van der Gucht, Interfacial tension between a complex coacervate phase and its coexisting aqueous phase, Soft Matter, 2010, 6, 172-178.

[10] D. Priftis, R. Farina and M. Tirrell, Interfacial energy of polypeptide complex coacervates measured via capillary adhesion, Langmuir, 2012, 28, 8721-8729.

[11] J. Quin, D. Priftis, R. Farina, S.L. Perry, L. Leon, J. Whitmer, K. Hoffmann, M. Tirrell and J.J de Pablo, Interfacial tension of polyelectrolyte complex coacervate phases, ACS Macro Lett., 2014, 3, 565-568. 
[12] N. Devi, M. Sarmah, B. Khatun and T.K. Maji, Encapsulation of active ingredients in polysaccharide-protein complex coacervates, Adv. Colloid Interface Sci., 2017, 239, 136-145.

[13] G. Dardelle and P. Erni, Three-phase interactions and interfacial transport phenomena in coacervate/oil/water systems, Adv. Colloid Interface Sci., 2014, 206, 79-91.

[14] C. Thies, Encapsulation and controlled release technologies in food systems, ed. J.M. Lakkis, Wiley Blackwell, Oxford, $2^{\text {nd }}$ edition, 2016, Ch. 3.

[15] S. Torza and S.G. Mason, Three-phase interactions in shear and electrical fields, J. Colloid Interface Sci., 1970, 33, 67-83.

[16] Modern Aspects of Emulsion Science, ed. B.P. Binks, The Royal Society of Chemistry, Cambridge, 1998, pp. 1-48.

[17] I.D. Morrison and S. Ross, Colloidal Dispersions. Suspensions, Emulsions, and Foams, Wiley, New York, 2002.

[18] M. Evans, I. Ratcliffe and P.A. Williams, Emulsion stabilisation using polysaccharideprotein complexes, Curr. Opin. Colloid Interface Sci., 2013, 18, 272-282.

[19] L. Jourdain, M.E. Leser, C. Schmitt, M. Michel and E. Dickinson, Stability of emulsions containing sodium caseinate and dextran sulfate: Relationship to complexation in solution, Food Hydrocolloids, 2008, 22, 647-659.

[20] A. K. Stone and M. T. Nickerson, Formation and functionality of whey protein isolate(kappa-, iota-, and lambda-type) carrageenan electrostatic complexes, Food Hydrocolloids, 2012, 27, 271-277.

[21] K. G. Zinoviadou, E. Scholten, T. Moschakis and C. G. Biliaderis, Properties of emulsions stabilised by sodium caseinate-chitosan complexes, Int. Dairy J., 2012, 26, 94-101.

[22] A.M. Bago Rodriguez, B.P. Binks and T. Sekine, Novel stabilisation of emulsions by soft particles: polyelectrolyte complexes, Faraday Discuss., 2016, 191, 255-285.

[23] D.E. Koppel, Analysis of macromolecular polydispersity in intensity correlation spectroscopy: the method of cumulants, J. Chem. Phys., 1972, 57, 4814-4820.

[24] M. Smoluchowski, in Handbuch der Electrizität und des Magnetismus, Leipzig, 1921, Vol. 2, p. 366. 
[25] W.D. Harkins and H.F. Jordan, A method for the determination of surface and interfacial tension from the maximum pull on a ring, J. Am. Chem. Soc., 1930, 52, 1751-1772.

[26] J.H. Clint, Adhesion and components of solid surface energies, Curr. Opin. Colloid Interface Sci., 2001, 6, 28-33.

[27] B.P. Binks, T. Sekine and A.T. Tyowua, Dry oil powders and oil foams stabilised by fluorinated clay platelet particles, Soft Matter, 2014, 10, 578-589.

[28] J. Choi and M.F. Rubner, Influence of the degree of ionisation on weak polyelectrolyte multilayer assembly, Macromolecules, 2005, 38, 116-124.

[29] S.E. Burke and C.J. Barrett, Acid-base equilibria of weak polyelectrolytes in multilayer thin films, Langmuir, 2003, 19, 3297-3303.

[30] G.S. Manning. Limiting laws and counterion condensation in polyelectrolyte solutions I. Colligative properties, J. Chem. Phys., 1969, 51, 924-933.

[31] A.I. Petrov, A.A. Antipov and G.B. Sukhorukov, Base-acid equilibria in polyelectrolyte systems: From weak polyelectrolytes to interpolyelectrolyte complexes and multilayered polyelectrolyte shells, Macromolecules, 2003, 36, 10079-10086.

[32] L. Vitorazi, N. Ould-Moussa, S. Sekar, J. Fresnais, W. Loh, J.-P. Chapel and J.-F. Berret. Evidence of two-step process and pathway dependency in the thermodynamics of poly(diallyldimethylammonium chloride)/poly(sodium acrylate) complexation, Soft Matter, 2014, 10, 9496-9505.

[33] J. Požar and D. Kovačević, Complexation between polyallylammonium cations and polystyrenesulfonate anions: the effect of ionic strength and the electrolyte type, Soft Matter, 2014, 10, 6530-6545.

[34] J. Koetz and S. Kosmella, Polyelectrolytes and Nanoparticles, Springer-Verlag, Berlin, 2007, p. 36.

[35] T. Alonso, J. Irigoyen, J.J. Iturri, I.L. Iarena and S.E. Moya, Study of the multilayer assembly and complex formation of poly(diallyldimethylammonium chloride) (PDADMAC) and poly(acrylic acid) (PAA) as a function of pH, Soft Matter, 2013, 9, 1920-1928. 
[36] V.S. Meka, M.K.G. Sing, M.R. Pichika, S.R. Nali, V.R.M. Kolapalli and P. Kesharwani, A comprehensive review on polyelectrolyte complexes, Drug Discovery Today, 2017, 22, 1697-1706.

[37] V. Starchenko, M. Müller and N. Lebovka, Sizing of PDADMAC/PSS complex aggregates by polyelectrolyte and salt concentration and PSS molecular weight, J. Phys. Chem. $B, 2012,116,14961-14967$.

[38] J. Koetz and S. Kosmella, Interactions between poly(diallyldimethylammonium chloride) and poly(acrylic acid) in dependence on polymer concentration, presented in part at the International Conference on Scaling Concepts and Complex Fluids, Italy, 1994.

[39] S. Nimesh, A. Saxena, A. Kumar and R. Chandra, Improved transfection efficiency of chitosan-DNA complexes employing reverse transfection, J. Appl. Polym. Sci., 2012, 124, $1771-1777$.

[40] D.S. Williams, S. Koga, C. Rohaida, C. Hak, A. Majrekar, A.J. Patil, A.W. Perriman and S. Mann, Polymer/nucleotide droplets as bio-inspired functional micro-compartments, Soft Matter, 2012, 8, 6004-6014.

[41] Y. Ishimuro and K. Ueberreiter, The surface tension of poly(acrylic acid) in aqueous solution. Colloid Polym. Sci., 1980, 258, 928-931.

[42] A. Forgiarini, J. Esquena, C. González and C. Solans, Formation of nano-emulsions by low-energy emulsification methods at constant temperature, Langmuir, 2001, 17, 2076-2083.

[43] A.S. Prata and C.R.F. Grosso, Influence of the oil phase on the microencapsulation by complex coacervation, J. Am. Oil Chem. Soc., 2015, 92, 1063-1072.

[44] N.R. Pallas and Y. Harrison. An automated drop shape apparatus and the surface tension of pure water, Colloids Surf., 1990, 43, 169-194.

[45] B.P. Binks and J.H. Clint, Solid wettability from surface energy components: Relevance to Pickering emulsions, Langmuir, 2002, 18, 1270-1273.

[46] J.H. Clint and A.C. Wicks, Adhesion under water: surface energy considerations, Int. J. Adhes. Adhes., 2001, 21, 267-273. 
[47] A. Loxley and B. Vincent, Preparation of poly(methylmethacrylate) microcapsules with liquid cores, J. Colloid Interface Sci., 1998, 208, 49-62.

[48] A.L. Tasker, J.P. Hitchcock, L. He, E.A. Baxter, S. Biggs and O.J. Cayre, The effect of surfactant chain length on the morphology of poly(methylmethacrylate) microcapsules for fragrance oil encapsulation, J. Colloid Interface Sci., 2016, 484, 10-16. 
Table 1. Molecular characteristics of polyelectrolytes and chemical structure, source, purity and density $\left(20^{\circ} \mathrm{C}\right)$ of all the oils used.

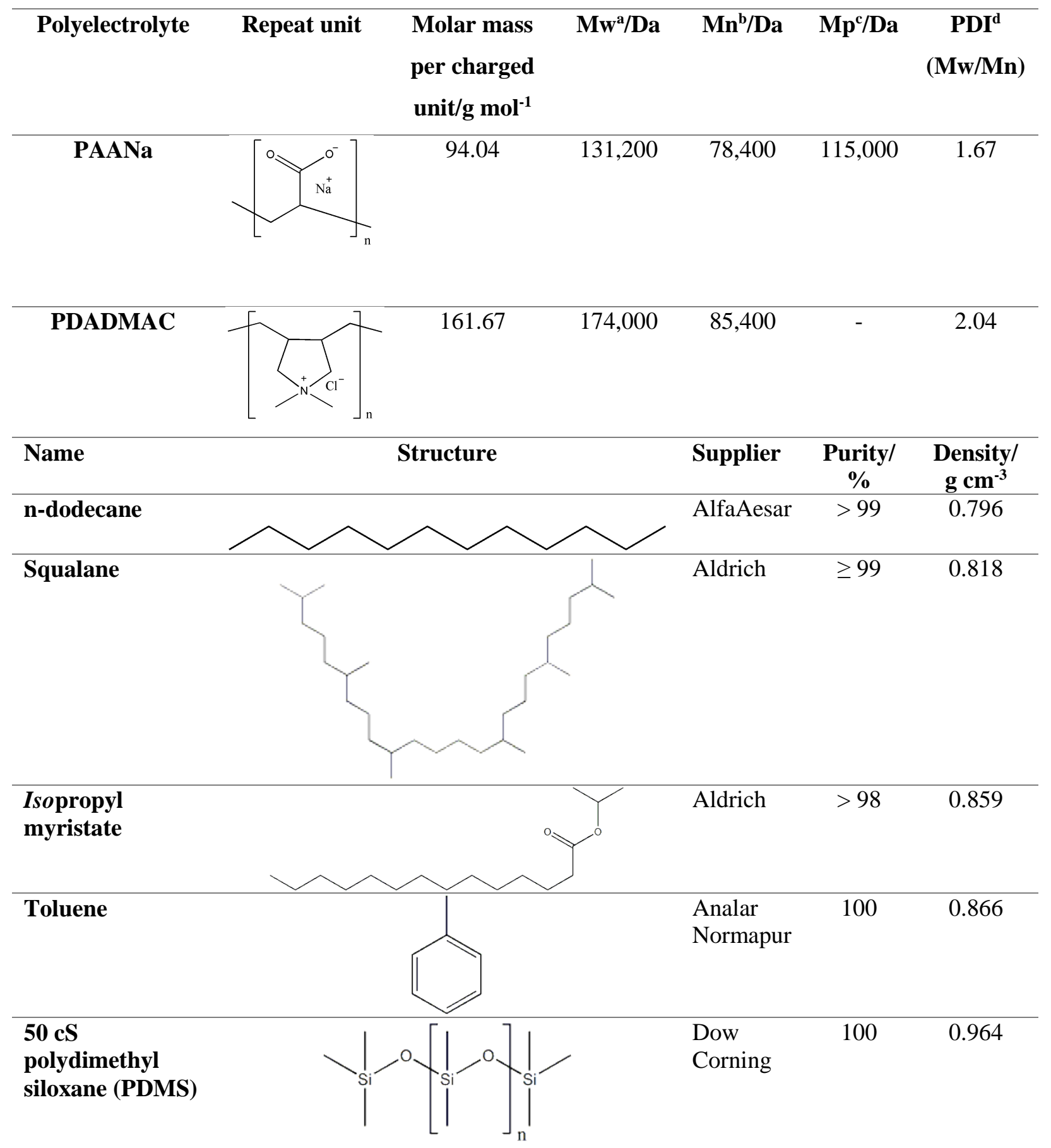

${ }^{\mathrm{a}} \mathrm{Mw}$ : weight average molecular weight; ${ }^{\mathrm{b}} \mathrm{Mn}$ : number average molecular weight; ${ }^{\mathrm{c}} \mathrm{Mp}$ : molar mass at the peak maximum; ${ }^{\mathrm{d}} \mathrm{PDI}$ : polydispersity index 
Table 2. Measured surface and interfacial tensions for the different oils $\left(\gamma_{a o}, \gamma_{o w}\right)$ and calculated surface energy of the oil-coacervate interface, $\gamma_{o c}$. Calculated spreading coefficients $\mathrm{S}$ for the different scenarios together with the predicted and observed morphology of oil droplets in water in the presence of coacervate phase.

\begin{tabular}{|c|c|c|c|c|c|c|c|c|}
\hline Oil & $\gamma_{o w} / \mathrm{mN} \mathrm{m}^{-1}$ & $\gamma_{a 0} / \mathrm{mN} \mathrm{m}^{-1}$ & $\gamma_{o c} / \mathrm{mN} \mathrm{m}^{-1}$ & $\mathrm{~S}_{1} / \mathbf{m N ~ m}^{-1}$ & $\mathrm{~S}_{2} / \mathrm{mN} \mathrm{m}^{-1}$ & $\mathrm{~S}_{3} / \mathrm{mN} \mathrm{m}^{-1}$ & Predicted & Observed \\
\hline Dodecane & $52.5 \pm 2.0$ & $24.7 \pm 0.3$ & $26.2 \pm 0.8$ & $-74.5 \pm 6.1$ & $-30.5 \pm 2.5$ & $22.8 \pm 1.8$ & $\begin{array}{l}\text { Complete } \\
\text { engulfing }\end{array}$ & $\begin{array}{l}\text { Complete } \\
\text { engulfing }\end{array}$ \\
\hline Toluene & $36.1 \pm 1.4$ & $28.0 \pm 0.1$ & $22.9 \pm 0.6$ & $-54.8 \pm 4.5$ & $-17.4 \pm 1.4$ & $9.0 \pm 0.7$ & $\begin{array}{l}\text { Complete } \\
\text { engulfing }\end{array}$ & $\begin{array}{l}\text { Complete } \\
\text { engulfing }\end{array}$ \\
\hline $\begin{array}{l}\text { Isopropyl } \\
\text { myristate }\end{array}$ & $28.8 \pm 0.7$ & $28.2 \pm 0.1$ & $22.7 \pm 0.6$ & $-47.3 \pm 3.6$ & $-10.3 \pm 0.8$ & $1.89 \pm 0.1$ & $\begin{array}{l}\text { Complete } \\
\text { engulfing }\end{array}$ & $\begin{array}{c}\text { Non- } \\
\text { engulfing }\end{array}$ \\
\hline Squalane & $52.5 \pm 0.6$ & $28.3 \pm 0.1$ & $22.6 \pm 0.6$ & $-70.9 \pm 5.1$ & $-34.1 \pm 2.5$ & $25.7 \pm 1.9$ & $\begin{array}{l}\text { Complete } \\
\text { engulfing }\end{array}$ & $\begin{array}{c}\text { Non- } \\
\text { engulfing }\end{array}$ \\
\hline PDMS & $50.5 \pm 0.8$ & $20.4 \pm 0.1$ & $30.5 \pm 0.8$ & $-76.8 \pm 5.6$ & $-24.2 \pm 1.7$ & $15.8 \pm 1.1$ & $\begin{array}{l}\text { Complete } \\
\text { engulfing }\end{array}$ & $\begin{array}{l}\text { Complete } \\
\text { engulfing }\end{array}$ \\
\hline
\end{tabular}


Table 3. Values of dispersion component $\left(\gamma_{a l}^{d}\right)$, polar component $\left(\gamma_{a l}^{p}\right)$ and $\gamma_{a l}$ for the test liquids used at $25{ }^{\circ} \mathrm{C}$ (taken from ref. 46) and three-phase liquid-air contact angles measured through the liquid on the surface of a glass slide covered with coacervate phase.

\section{Tension $/ \mathrm{mN} \mathrm{m}^{-1}$}

\begin{tabular}{ccccc} 
& & & & $\boldsymbol{\theta}_{\boldsymbol{a l}}{ }^{\circ}$ \\
\hline Liquid & $\gamma_{\boldsymbol{a l}}^{\boldsymbol{d}}$ & $\boldsymbol{\gamma}_{\boldsymbol{a l}}^{\boldsymbol{p}}$ & $\boldsymbol{\gamma}_{\boldsymbol{a l l}}$ & \\
\hline Water & 21.8 & 51.0 & 72.8 & $50 \pm 3$ \\
\hline Glycerol & 34.0 & 30.0 & 64.0 & $55 \pm 9$ \\
\hline Formamide & 39.0 & 19.0 & 58.0 & $38 \pm 5$ \\
\hline $\boldsymbol{\alpha - B r o m o n a p h t h a l e n e}$ & 44.4 & 0.0 & 44.4 & $27 \pm 3$ \\
\hline Hexadecane & 27.8 & 0.0 & 27.8 & $<5$ \\
\hline
\end{tabular}


Figure 1. Appearance of freshly prepared aqueous PEC dispersions from $0.1 \mathrm{~g} \mathrm{~L}^{-1}$ individual PEL solutions at different XPAANa (given) and $\mathrm{pH}$. Scale bar $=1 \mathrm{~cm}$.

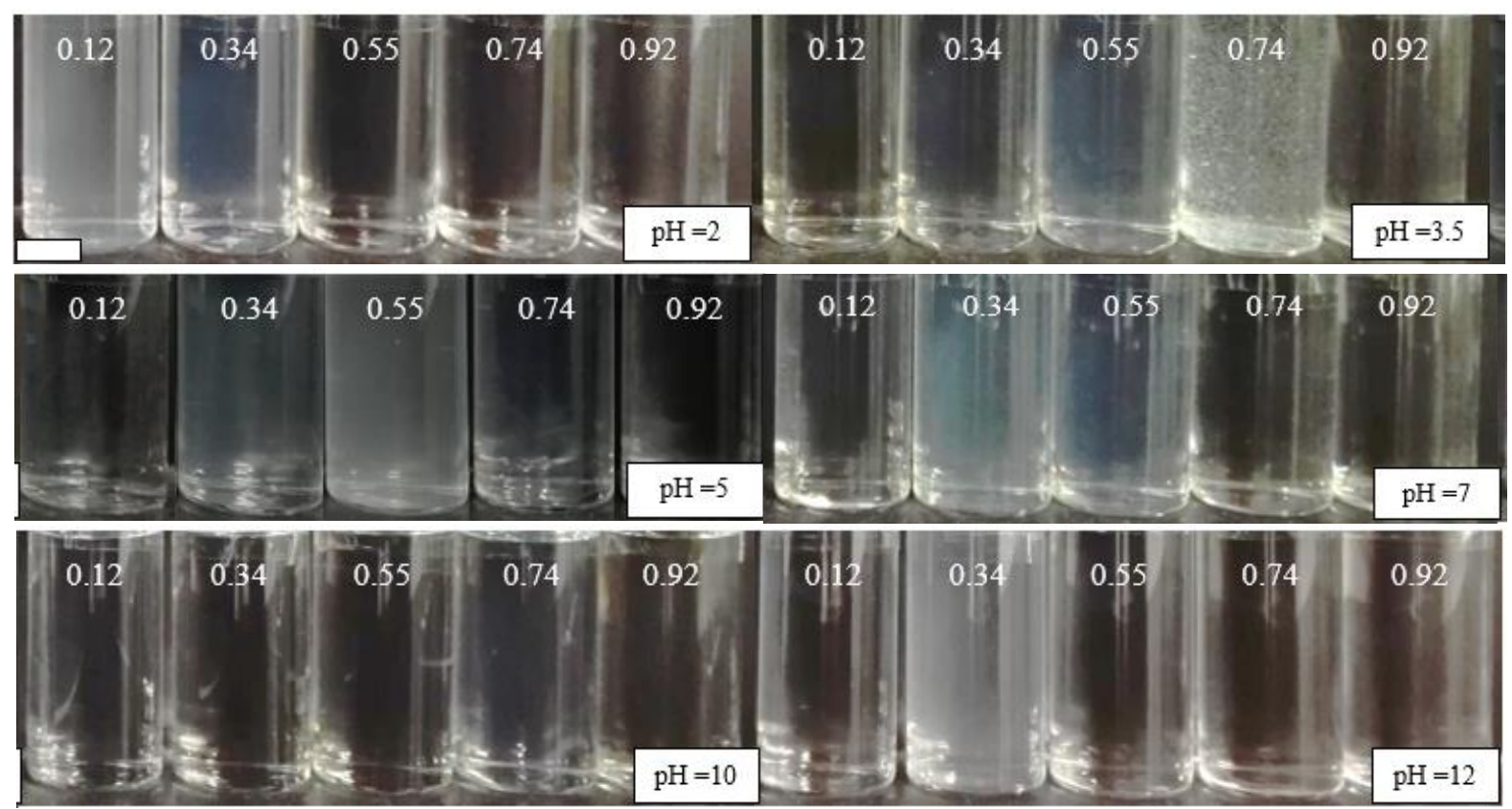


Figure 2. Variation of the zeta potential with XPAANa for aqueous PEC dispersions prepared from $0.1 \mathrm{~g} \mathrm{~L}^{-1}$ individual PEL solutions at different $\mathrm{pH}$ where PAANa is (a) uncharged, (b) progressively charged and (c) fully charged.

(a)

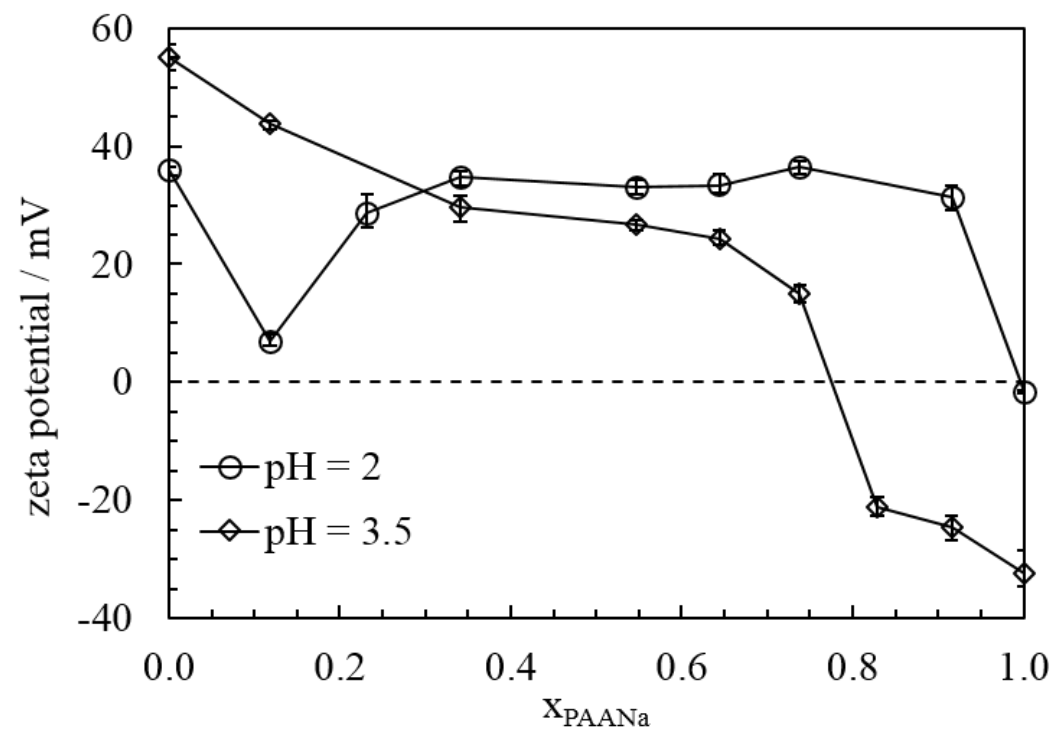

(b)

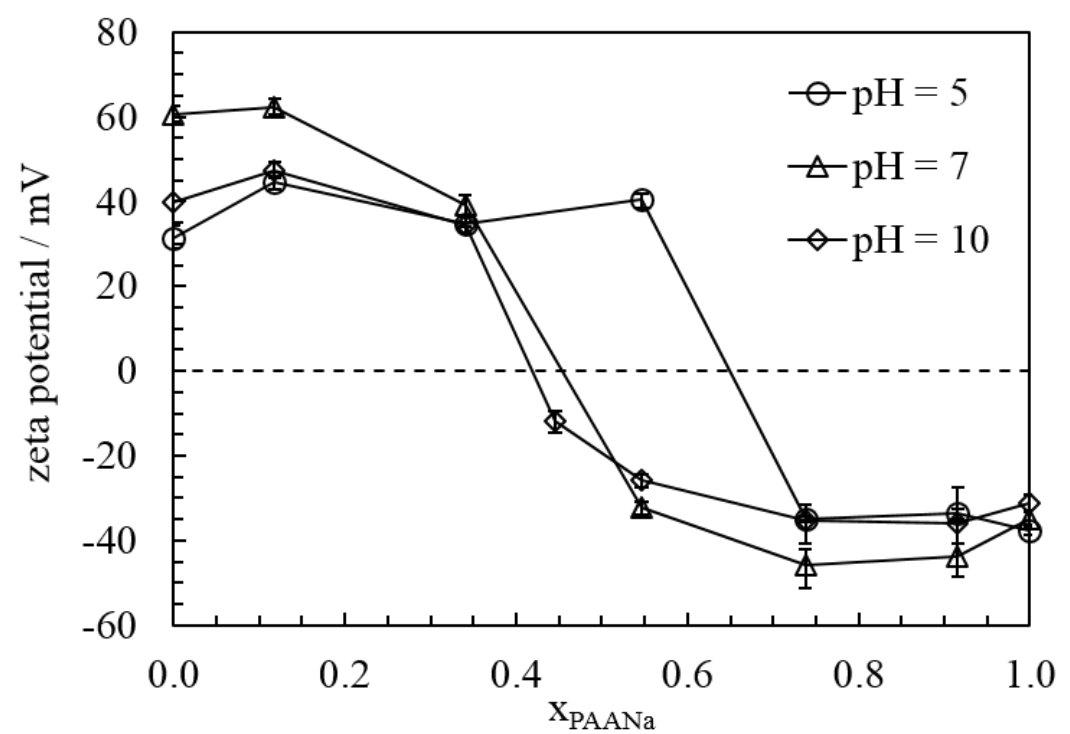

(c)

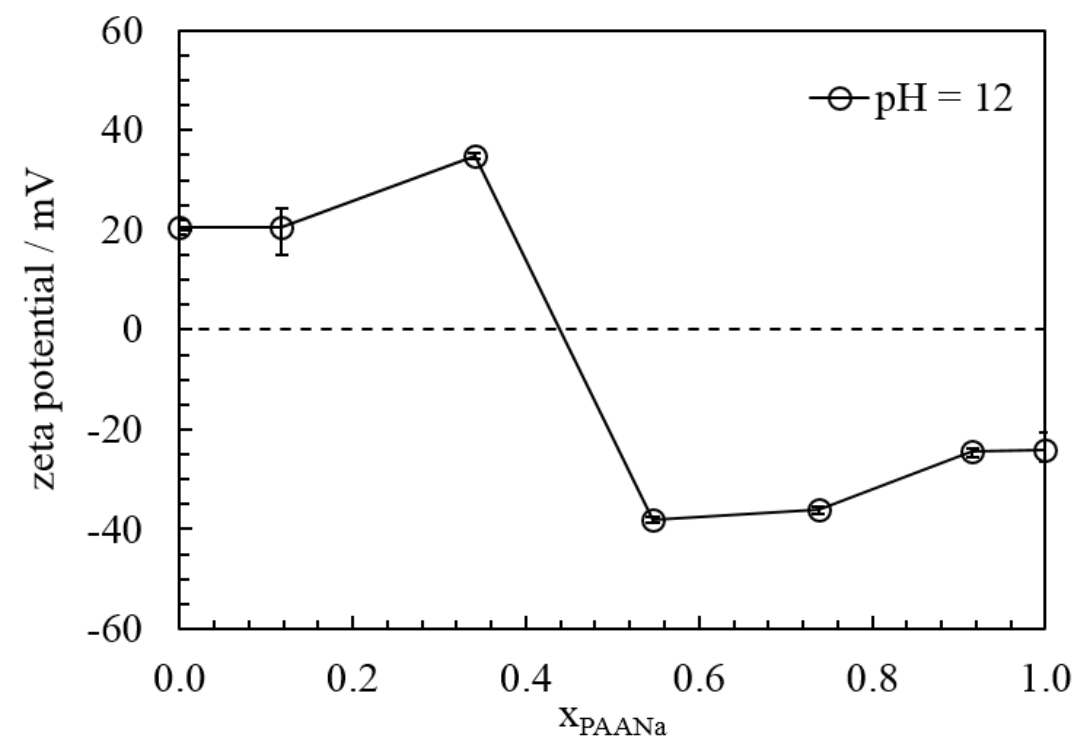


Figure 3. Variation of $\mathrm{x}_{\mathrm{PAANa}}$ at zeta potential $=0$ with $\mathrm{pH}$ for aqueous $\mathrm{PEC}$ dispersions prepared from $0.1 \mathrm{~g} \mathrm{~L}^{-1}$ individual PEL solutions.

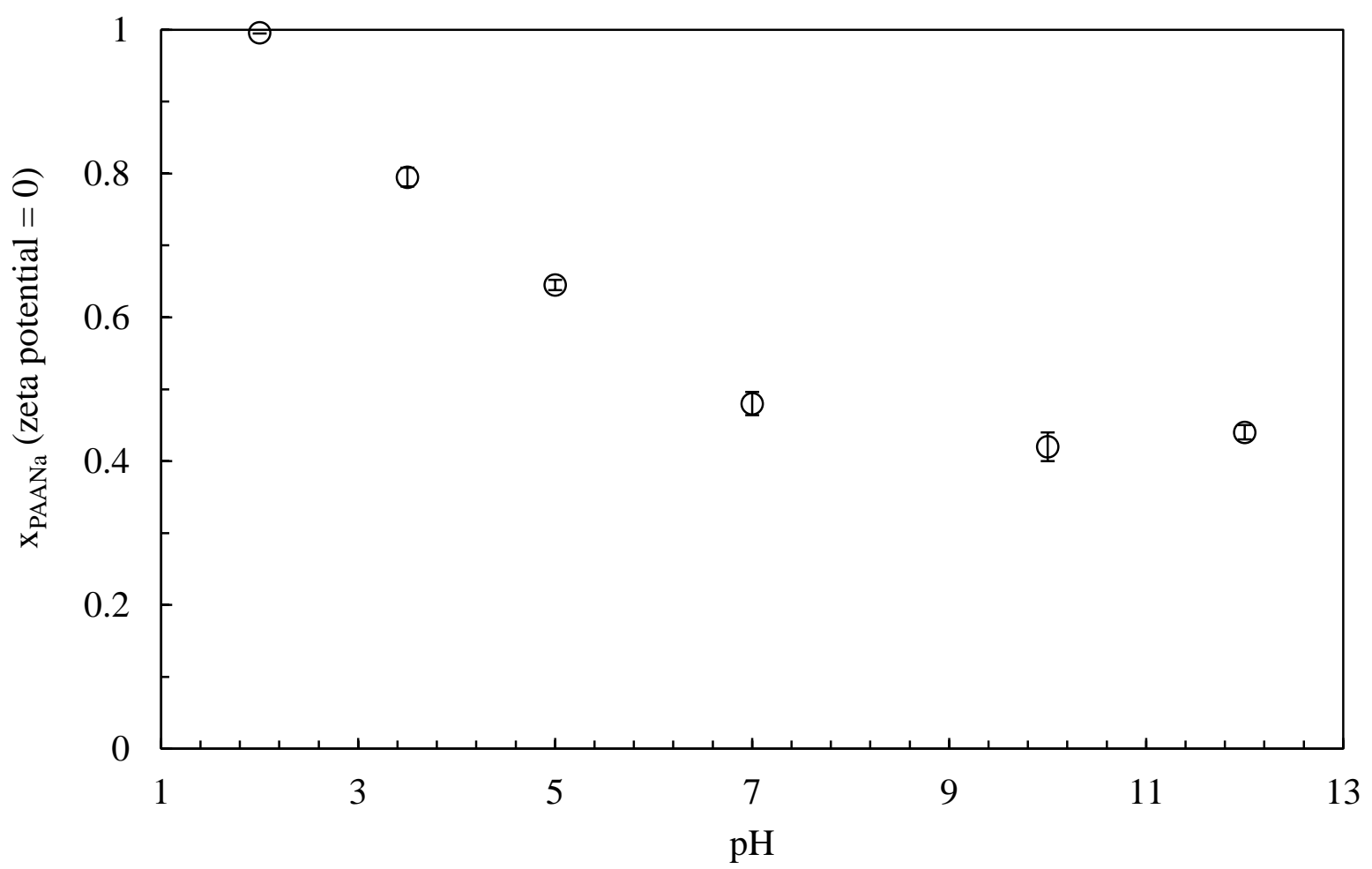


Figure 4. Appearance of fresh aqueous PEC dispersions prepared from $5 \mathrm{~g} \mathrm{~L}^{-1}$ PEL solutions at (a) $\mathrm{pH}=10$ and (b) $\mathrm{pH}=4$ at different XPAANa (given). Scale bars $=1 \mathrm{~cm}$. Optical microscope image of a drop of the dispersion at (c) $\mathrm{pH}=10, \mathrm{x}_{\mathrm{PAANa}}=0.57$ or $(\mathrm{d}) \mathrm{pH}=4, \mathrm{X}_{\text {PAANa }}=0.13$.
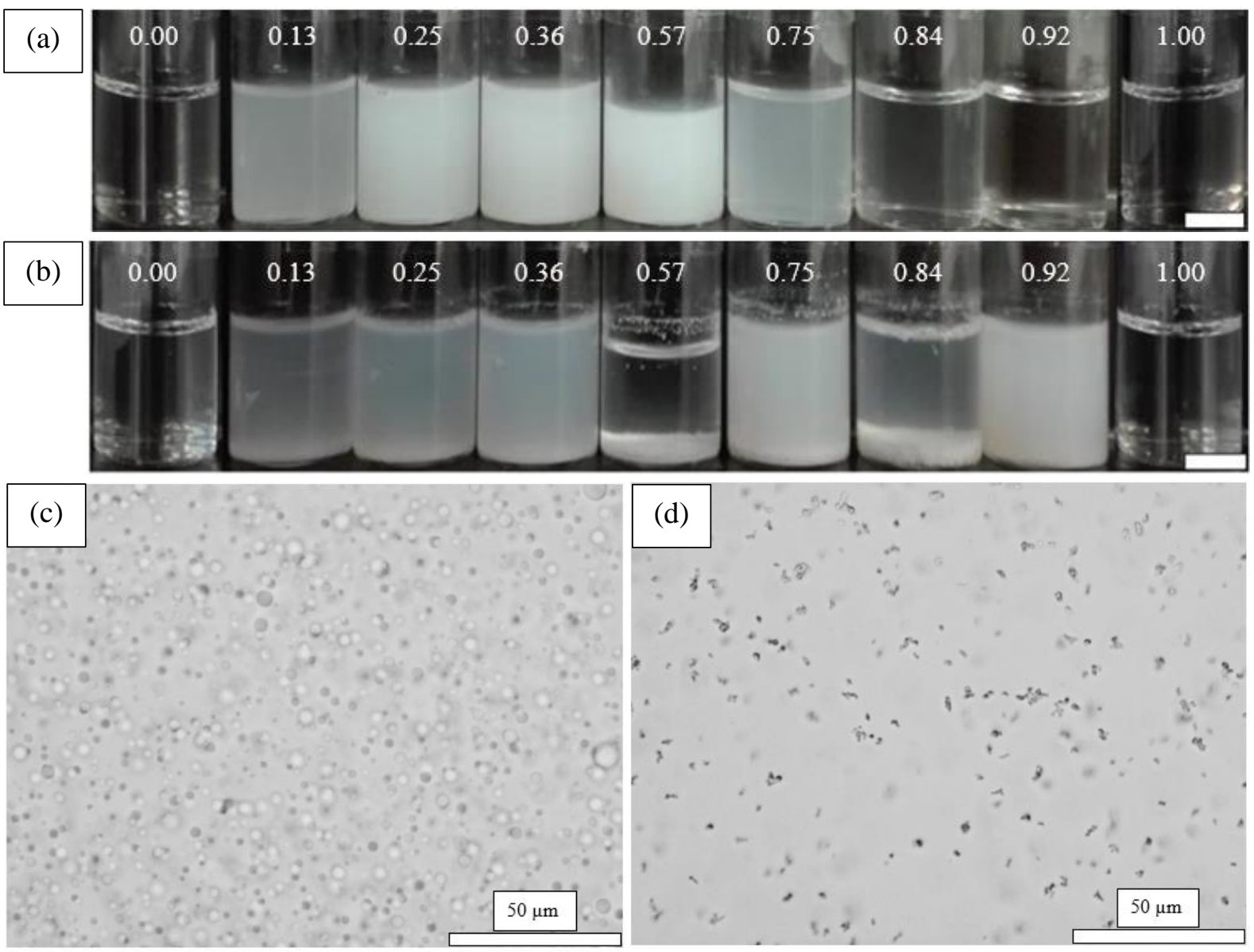
Figure 5. (1) Appearance of $1 \mathrm{~mL}$ of aqueous PEC dispersion ([PEL] $=10 \mathrm{~g} \mathrm{~L}^{-1}, \mathrm{pH}=10$, XPAANa $=0.5)$ before and after centrifugation. The three different phases separated after centrifugation are supernatant (phase A), interface (phase B) and coacervate (phase C). Optical microscope images of the different phases are included. For phase B, images from the same drop are shown at different times. (2) Appearance of $1 \mathrm{~mL}$ of aqueous PEC dispersion ([PEL] $=5 \mathrm{~g} \mathrm{~L}^{-1}, \mathrm{pH}=4$, XPAANa $\left.=0.5\right)$ after centrifugation.

(1)

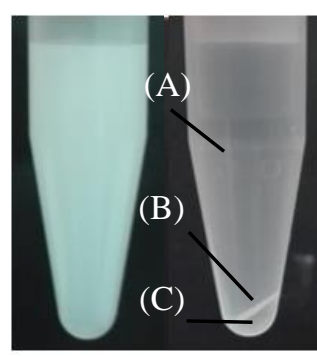

$\mathrm{t}=0$

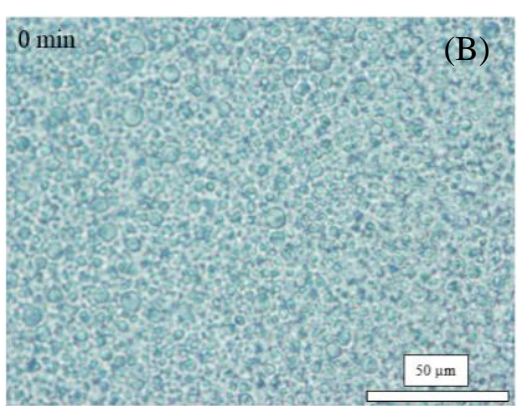

$(2)$

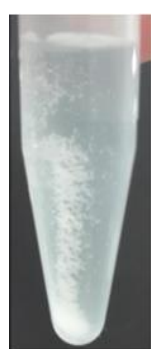

)
(A)
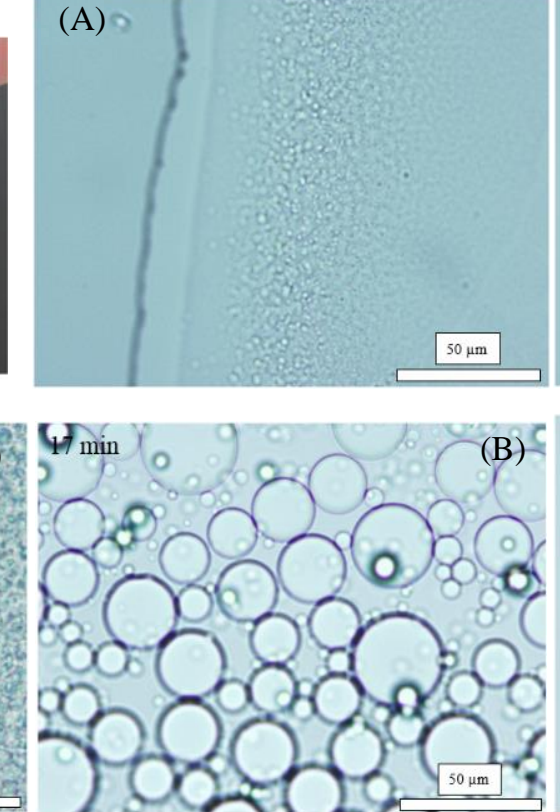

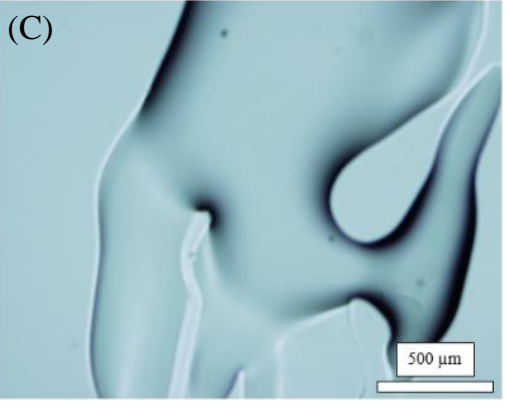

$22 \mathrm{~min}$

$50 \mu \mathrm{m}$ 
Figure 6. (a) Evolution of the stability of aqueous PEC dispersions prepared from $5 \mathrm{~g} \mathrm{~L}^{-1}$ individual PEL solutions ( $\mathrm{XPAANa} \approx 0.50)$ at different $\mathrm{pH}$ (given). Scale bar $=1 \mathrm{~cm}$. Optical microscope images of the aqueous PEC dispersions at pH (b) 2, (c) 3, (d) 4 and (e) 6.

(a) Coacervate Coacervate/precipitate

\section{Coacervate}

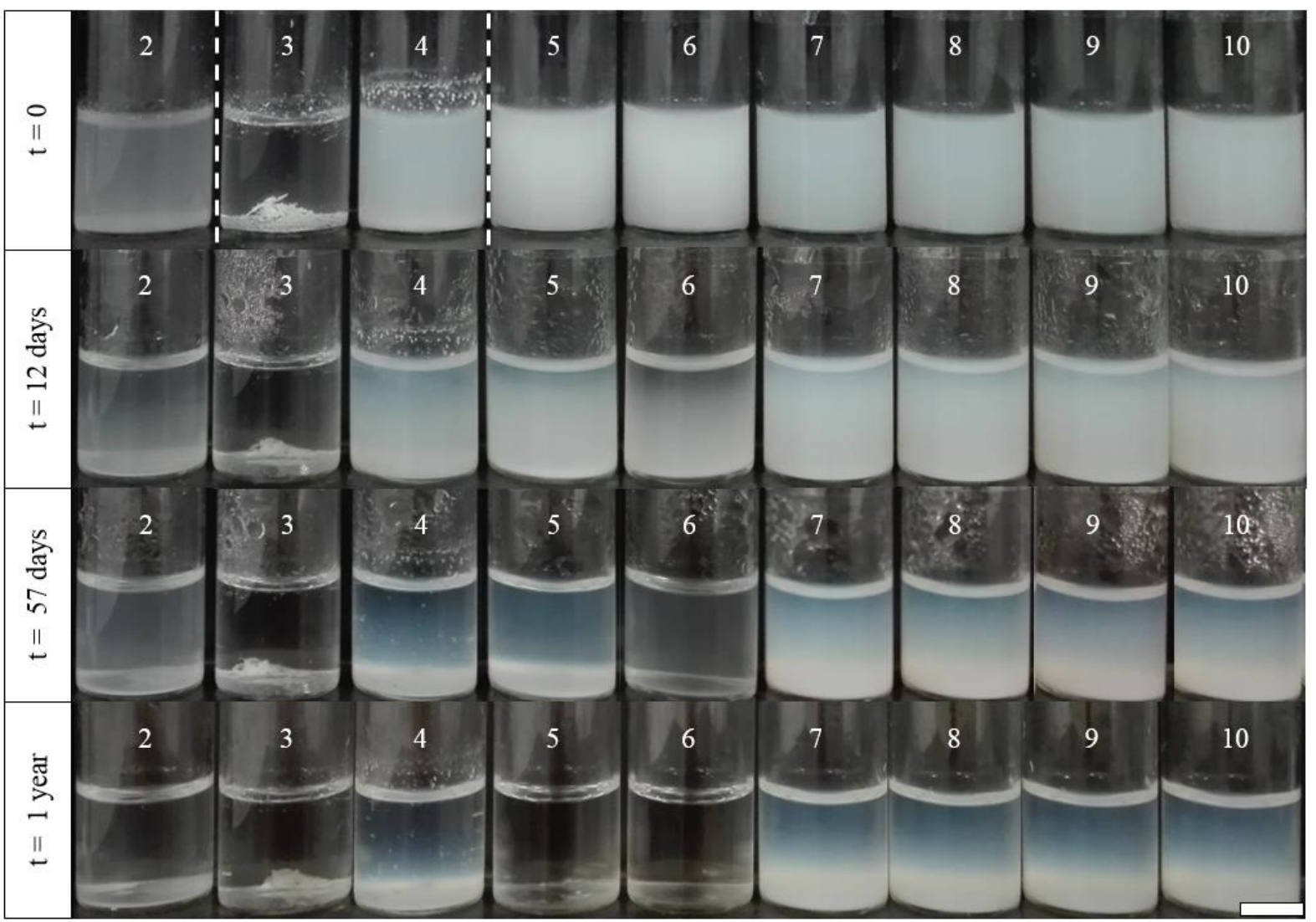

(b)

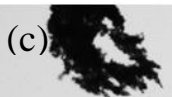

$50 \mu \mathrm{m}$
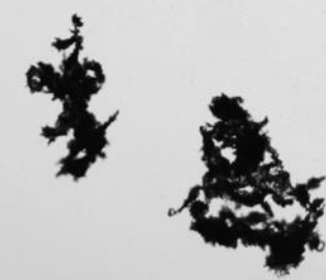

$500 \mu \mathrm{m}$
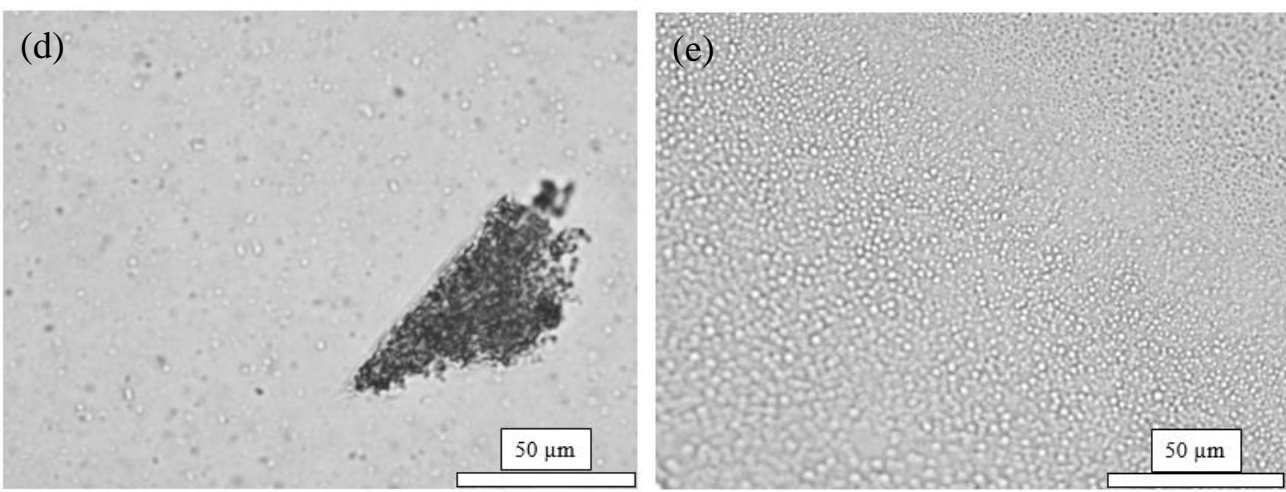
Figure 7. TEM images of fresh aqueous PEC dispersion (XPAANa $=0.5)$ prepared from $5 \mathrm{~g} \mathrm{~L}^{-1}$ PEL solutions at $\mathrm{pH}=10$ at different magnifications. (a), (c) and (d) correspond to different regions of the grid, (b) is a higher magnification image of (a). All correspond to the unstained sample.

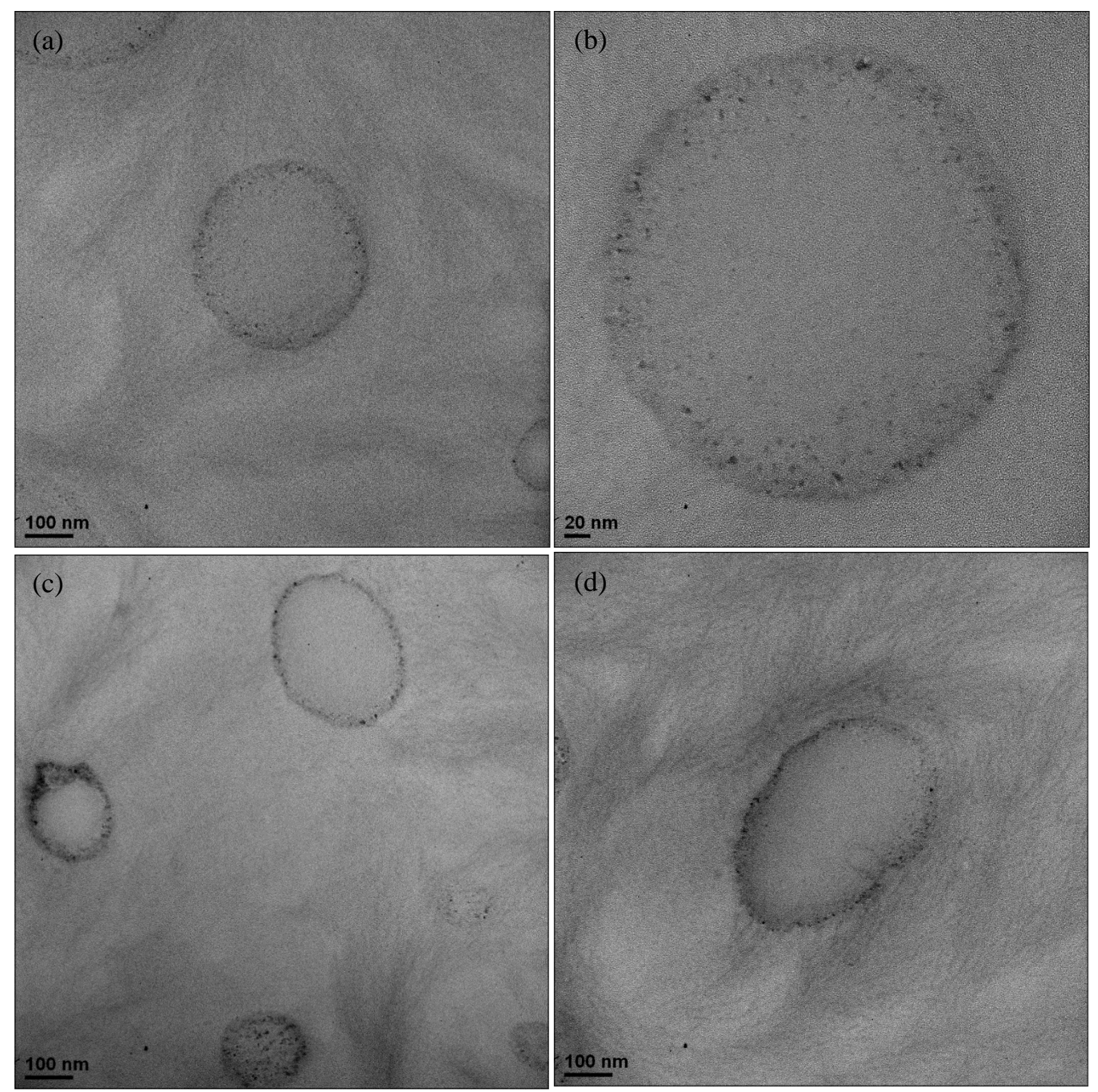


Figure 8. Stability with time of dodecane-in-water $\left(\phi_{o}=0.20\right)$ emulsions prepared from aqueous PEC dispersions ([PEL] $=5 \mathrm{~g} \mathrm{~L}^{-1}, \mathrm{pH}=10$, XPAANa $=0.5$ ). (a) Oil added in one step, (b) oil added stepwise; $\phi_{o}$ in the overall emulsion after each addition is given. Scale bar $=1 \mathrm{~cm}$.

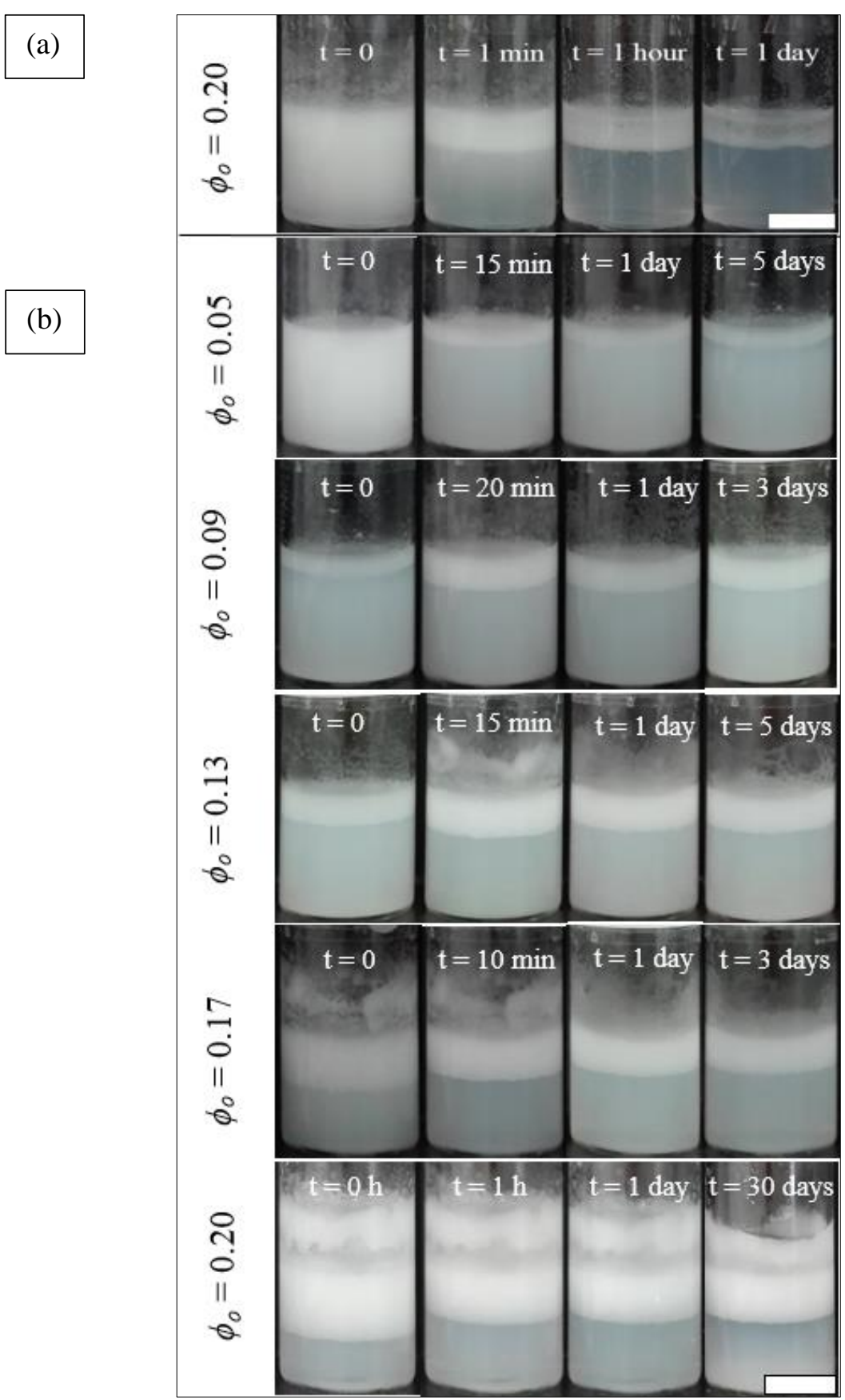


Figure 9. (a) Optical microscope images of freshly prepared dodecane-in-water emulsion $\left(\phi_{o}\right.$ $=0.05)$ from aqueous PEC dispersion $\left([\mathrm{PEL}]=5 \mathrm{~g} \mathrm{~L}^{-1}, \mathrm{pH}=10\right.$, XPAANa $\left.=0.5\right)$ at different magnifications (given). (b) Cryo-SEM images of freshly prepared dodecane-in-water emulsion $\left(\phi_{o}=0.20\right)$ from aqueous PEC dispersion $\left([\mathrm{PEL}]=5 \mathrm{~g} \mathrm{~L}^{-1}, \mathrm{pH}=10, \mathrm{XPAANa}=0.5\right)$ at different magnifications (given). In both cases, the emulsion was prepared with the addition of oil stepwise.

(a)

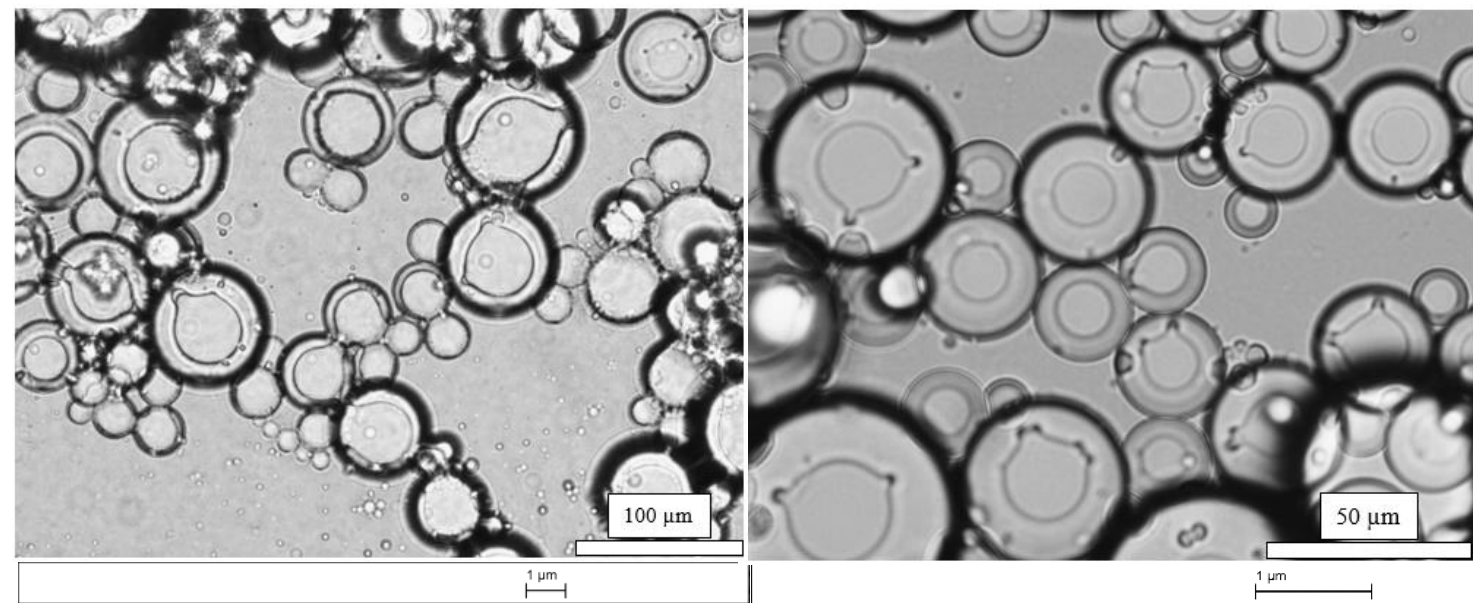

(b)

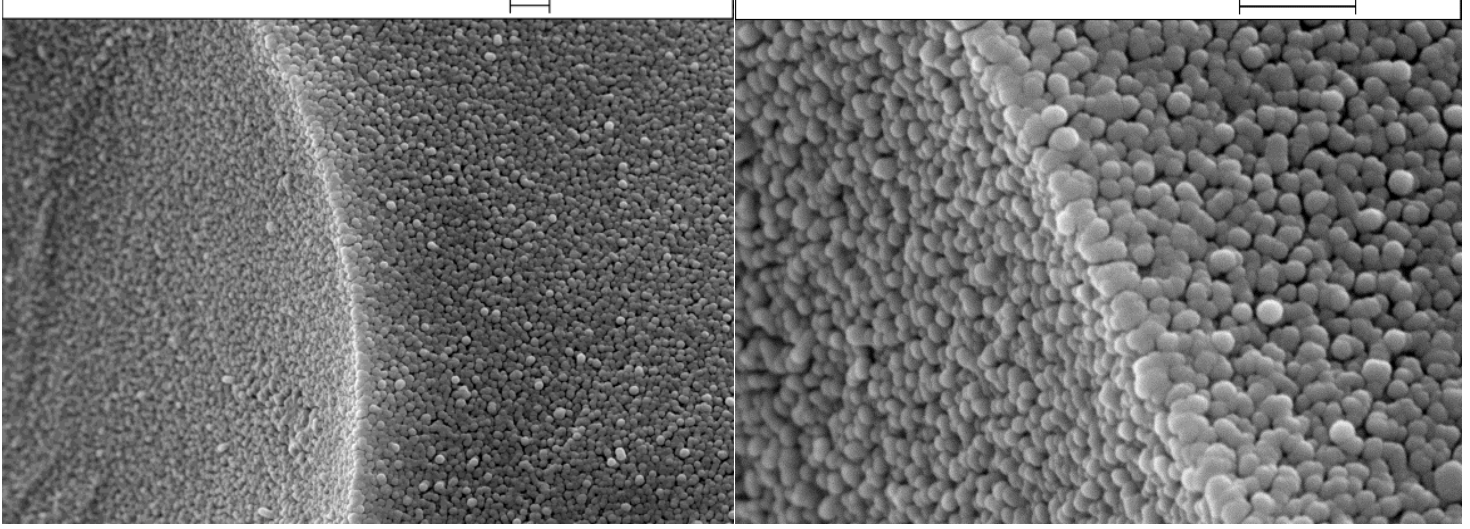


Figure 10. 3-D surface energy plot for the coacervate phase as a function of the possible values of $\gamma_{a c}^{d}$ and $\gamma_{a c}^{p}$. The ordinate represents the goodness of fit to contact angles.

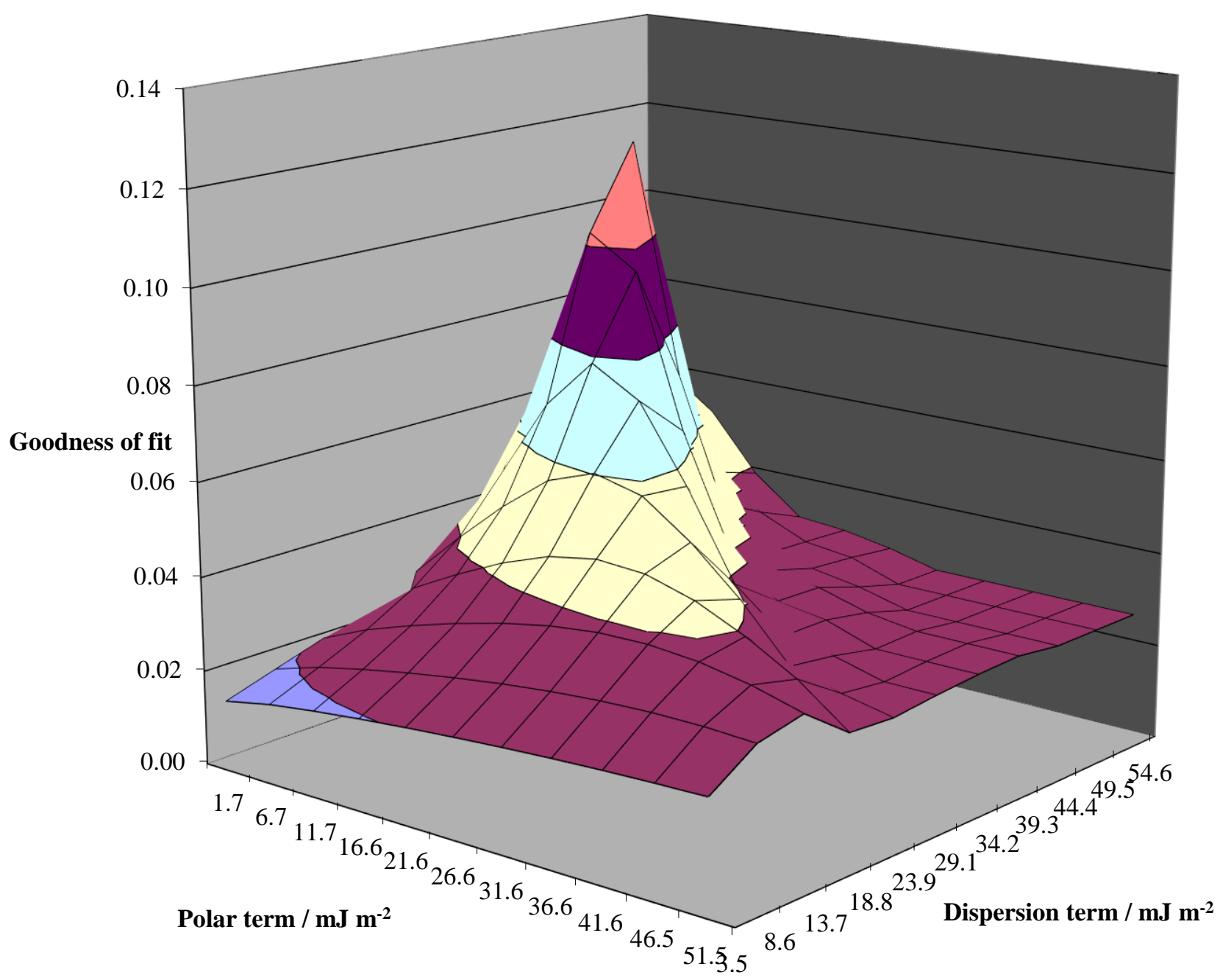

